Proceedings of the Edinburgh Mathematical Society (2003) 46, 719-745 (C)

DOI:10.1017/S0013091502001141 Printed in the United Kingdom

\title{
CAPACITIES AND JACOBI MATRICES
}

\author{
AHMED SEBBAR ${ }^{1}$ AND THÉRÈSE FALLIERO ${ }^{2}$ \\ ${ }^{1}$ UFR Mathématiques et Informatique, Université Bordeaux I, \\ 33405 Talence Cedex, France (sebbar@math.u-bordeaux.fr) \\ ${ }^{2}$ Faculté des Sciences, Université d'Avignon, 84000 Avignon, \\ France (Therese.Falliero@univ-avignon.fr)
}

(Received 4 December 2002)

\begin{abstract}
In this paper, we use the theorem of Burchnall and Shaundy to give the capacity of the spectrum $\sigma(A)$ of a periodic tridiagonal and symmetric matrix. A special family of Chebyshev polynomials of $\sigma(A)$ is also given. In addition, the inverse problem is considered: given a finite union $E$ of closed intervals, we study the conditions for a Jacobi matrix $A$ to exist satisfying $\sigma(A)=E$. We relate this question to Carathéodory theorems on conformal mappings.
\end{abstract}

Keywords: Jacobi matrices; capacities; Burchnall-Shaundy Theorem; Carathéodory Kernel Theorem

2000 Mathematics subject classification: Primary 31B15; 30C20; 39A70

\section{Introduction}

In this work, a close connection is established between capacities of unions of closed intervals and Jacobi matrices. The links between the apparently distant themes rely on the role played by the Green function for multiply connected domains and their critical points.

Let $E$ be a finite union of $n=g+1$ closed intervals of the real line, $E=\left[E_{1}, E_{2}\right] \cup$ $\left[E_{3}, E_{4}\right] \cup \cdots \cup\left[E_{2 n-1}, E_{2 n}\right]$, the capacity $\mathcal{C}(E)$ of $E$ is given in the constant term of the expansion near $\infty$ by

$$
\mathcal{G}(z)=\log |z|-\log \mathcal{C}(E)+o(1),
$$

where $\mathcal{G}(z)$ is the Green function of the complement of $E$ in the Riemann sphere $\hat{\mathbb{C}}$ with pole at infinity. It soon becomes clear that there are some fundamental principles behind the question of the evaluation of $\mathcal{C}(E)$. We wish to provide several approaches for this evaluation and to establish connections with other problems and topics.

The capacity of an interval $[a, b]$ is one-quarter of its length and if we scale it to the interval $[-2,2]$, we can observe that the theory of the Chebyshev polynomials $T_{n}(X)$ with $T_{n}(2 \cos \theta)=2 \cos (n \theta), n \in \mathbb{N}$, is a part of the spectral analysis of the matrix $A=\left(a_{i, j}\right)_{1 \leqslant i, j}, a_{i, i+1}=a_{i, i-1}=1$ and $a_{i, j}=0$ otherwise. The matrix $A$ is tridiagonal, symmetric and of period one. The capacity of $[-2,2]$ is exactly the value of its nonvanishing entry. One of our objectives here is to develop a similar approach in the case 
of several intervals. First of all, in the case of two intervals, the capacity of $E$ was given by Achieser in $[\mathbf{1}, \mathbf{2}]$. Achieser used a conformal mapping of $\hat{\mathbb{C}} \backslash E$ into an annulus $A_{r, 1}=\{r<|z|<1\}$, where $r$ is the modulus of the doubly connected domain $\hat{\mathbb{C}} \backslash E$. The final result is formulated in terms of Jacobi theta functions, but no relation to the spectral theory is involved. We developed in [11] a method for the configuration of three intervals of the real line. However, our analysis is different from Achieser's, because no explicit conformal mapping is available for the triply connected domain $\hat{\mathbb{C}} \backslash E$. We use the theory of functions on the hyperelliptic curve associated with the equation

$$
y^{2}=\left(z-E_{1}\right)\left(z-E_{2}\right)\left(z-E_{3}\right)\left(z-E_{4}\right)\left(z-E_{5}\right)\left(z-E_{6}\right) .
$$

This is a genus-two situation and, by means of Rosenhain's relations, our formula for the capacity $\mathcal{C}(E)$ is quite similar to Achieser's and we recover it when one interval collapses to a point or when $E$ possesses some symmetry. In the last case, we have to reduce hyperelliptic integrals to elliptic integrals. It turns out that Rosenhain's relations are specific to the genus-two case. It is difficult to extend our method to get nice formulae in higher genus. Furthermore, no link to the spectral theory is involved in [11].

If $E$ is a compact set in the complex plane and if $M_{n}$ is the maximum absolute value on $E$ of the Chebyshev polynomial of degree $n$, then, by a transfer theorem of Fekete [12], the capacity of $E$ is $\mathcal{C}(E)=\lim _{n \rightarrow \infty} M_{n}^{1 / n}$. A very important observation is made by Robinson in his study of algebraic integers in real point sets in [29] and [30]. It says that if $E$ consists of a finite number of intervals and if for some positive integer $r$, the Chebyshev polynomial of degree $r$ oscillates exactly $r$ times between $\pm 2 \lambda$ on $E$, then, for any positive integer $n$, the polynomial $F_{n}(X)=\lambda^{n} T_{n}(f(X) / \lambda)$ is the Chebyshev polynomial of degree $n r$. Hence the capacity of $E$ is just $\mathcal{C}(E)=\lambda$. In other words, in order to compute the capacity of a set $E$, one needs to know only a monic polynomial of positive degree $r$ that oscillates exactly $r$ times between $\pm \lambda$ for a positive $\lambda$.

In this paper, we will explore the algebraic, the analytic and the geometric natures of this special polynomial for a given compact set of the real line of the form $E=$ $\left[E_{1}, E_{2}\right] \cup\left[E_{3}, E_{4}\right] \cup \cdots \cup\left[E_{2 n-1}, E_{2 n}\right]$. More precisely, we will start by looking at the direct problem. We first consider a Jacobi matrix $T=\left(a_{j, k}\right)_{j, k \in \mathbb{Z}}, a_{j, k}=0$ for $|j-k|>2$ and $a_{j, k} \neq 0$ for $|j-k|=1$ which is symmetric and $N$-periodic, that is $a_{i+N, j+N}=a_{i, j}$ for $i, j \in \mathbb{Z}$. The classical theorem of Burchnall and Chaundy asserts that if $P$ and $Q$ are two commuting differential operators of order $p$ and $q$, respectively, then they satisfy identically an algebraic relation of the form $F(P, Q)=0$. Here $F$ is of degree $q$ in $P$ and of degree $p$ in $Q$. We follow an idea of the almost forgotten paper by Nauman [21] for an early difference-operators version of the Burchnall-Chaundy Theorem to obtain an explicit polynomial $P(X) \in \mathbb{C}[X]$ of degree $N$ such that $P(T)=E_{N}$, the matrix defined by $e_{i, i+N}=e_{i, i-N}=1, e_{i, j}=0$ otherwise. By the Floquet theory, the spectrum $\sigma$ of $T$ will be shown to coincide with the inverse image of the interval $[-2,2]$ under the polynomial map $P(\lambda)=\mu$. We show by different arguments that the polynomial $P$ is, up to a multiplicative factor, the Chebyshev polynomial of degree $N$ of the compact set $E=\sigma=\sigma(T)$ and that it oscillates $N$ times on $\sigma$. From what we said above, this gives us the capacity $\mathcal{C}(\sigma)$. We will study the metric properties of 
the polynomial $P$ and the lemniscate $|P(\lambda)|=2$ to get bounds on the sizes of the components of $\sigma$. Furthermore, this polynomial will be connected to some universal differential equations. To deal with the inverse problem, we consider a given subset on the real line $E=\left[E_{1}, E_{2}\right] \cup\left[E_{3}, E_{4}\right] \cup \cdots \cup\left[E_{2 n-1}, E_{2 n}\right]$, and, following several authors (see [34] or $[\mathbf{1 7}, \mathbf{1 8}])$ in the study of the non-stationary Peierls model, we construct a Jacobi matrix $T$ with the spectrum $\sigma(T)$ equal to $E$. This problem has been extensively studied and the results are contained in several articles or books (see $[\mathbf{1 9}, \mathbf{3 2}]$ and the references therein). Our contribution here is to make this construction more geometric in the following sense: the constructed operator is always an almost-periodic difference operator in the sense of Bohr. In $[\mathbf{1 7}, \mathbf{1 8}]$, Krichever gave necessary and sufficient conditions on $T$ for it to be periodic. We will show that these conditions are similar to those considered by Robinson in his study of algebraic points in $[\mathbf{2 9}, \mathbf{3 0}]$. This idea will connect the problem of the approximation of almost-periodic difference operators by periodic difference operators to a continuity method for conformal mapping in the context of the Carathéodory Kernel Theorem.

Moreover, the operator $T$ depends on the choice of points $P_{1}, P_{2}, \ldots, P_{n-1}$ on the curve associated with the equation $y^{2}=\left(z-E_{1}\right)\left(z-E_{2}\right) \cdots\left(z-E_{2 n}\right)$ such that their projections $\gamma_{1}, \gamma_{2}, \ldots, \gamma_{n-1}$ are located in the gaps

$$
\gamma_{1} \in\left(E_{2}, E_{3}\right), \ldots, \quad \gamma_{n-1} \in\left(E_{2 n-2}, E_{2 n-1}\right) .
$$

The complex Green function with pole at infinity in $\hat{\mathbb{C}} \backslash E$ is given by the abelian integral $[\mathbf{1 1}, \mathbf{3 8}]$ :

$$
G(z)=\int_{E_{2 n}}^{z} h(\zeta) q(\zeta)^{-1 / 2} \mathrm{~d} \zeta
$$

where $q(\zeta)=\left(\zeta-E_{1}\right)\left(\zeta-E_{2}\right) \cdots\left(\zeta-E_{2 n}\right)$ and $h(\zeta)$ is a monic polynomial of degree $n-1$ of the form $h(\zeta)=\zeta^{n-1}+h_{n-2} \zeta^{n-2}+\cdots+h_{0}$ with coefficients satisfying the following system of equations (a Jacobi inversion problem):

$$
\begin{gathered}
\sum_{0 \leqslant i \leqslant n-1} h_{i} \int_{E_{2 j}}^{E_{2 j+1}} \zeta^{i}|q(\zeta)|^{-1 / 2} \mathrm{~d} \zeta=0, \quad 1 \leqslant j \leqslant n-1, \\
h_{n-1}=1 .
\end{gathered}
$$

It can be seen that the roots $\gamma_{1}^{*}, \gamma_{2}^{*}, \ldots, \gamma_{n-1}^{*}$ of the polynomial $h(\zeta)$, which will be called equilibrium points throughout this paper, have the following distribution:

$$
E_{2}<\gamma_{1}^{*}<E_{3}<E_{4}<\gamma_{2}^{*}<\cdots<E_{2 n-2}<\gamma_{n-1}^{*}<E_{2 n-1} .
$$

This is just another version of the classical theorem of Gauss-Lucas. This fact will allow us to choose $\gamma_{j}=\gamma_{j}^{*}, 1 \leqslant j \leqslant n-1$, in the construction of the operator $T$. We obtain an interesting relation between the complex Green function of $\hat{\mathbb{C}} \backslash E$ and the Green function of the operator $T$ (resolvent). Different forms of the capacity will be given from this connection between the two Green functions. Finally, we will consider the case where everything can be worked out somewhat explicitly, namely when $E$ has two components, $E=\left[E_{1}, E_{2}\right] \cup\left[E_{3}, E_{4}\right]$. 


\section{Direct problems}

A generalized Jacobi matrix of order $2 m$ is an infinite matrix $T=\left(a_{j, k}\right)_{j, k \in \mathbb{Z}}, a_{j, k}=0$ for $|j-k|>2 m$ and $a_{j, k} \neq 0$ for $|j-k|=m$. We follow the presentation of [21] and the notation of $[\mathbf{3 4}]$, where a more general definition is introduced. We consider only the case where $T$ is symmetric and $N$-periodic, that is $a_{i+N, j+N}=a_{i, j}$ for $i, j \in \mathbb{Z}$. A difference operator $A$ associated with $T$ is defined in the following way.

Let $y$ be a vector $y={ }^{\mathrm{t}}\left(\ldots, y_{-1}, y_{0}, y_{1}, \ldots\right)$, and $D$ the shift operator acting on $y$ by $(D y)_{k}=y_{k+1}$. The operator $A$ is given by

$$
(A y)_{n}=\sum_{k=-m}^{k=m} a_{n, n+k} y_{n+k}=\left(\sum_{-m}^{m} a_{n, n+k} D^{k}\right) y_{n} .
$$

For each positive integer $n$, we introduce the matrix $E_{n}=\left(e_{i j}\right)_{i, j \in \mathbb{Z}}, e_{i j}=1$ if $|i-j|=n$ and $e_{i j}=0$ otherwise. In other words, $E_{n}=D^{n}+D^{-n}, e_{k, k-n}=e_{k, k+n}=1$, for $k \in \mathbb{Z}$.

\subsection{Periodic Jacobi matrices}

If $A$ is a generalized matrix of order $2 m$ and period $N$, then it is permutable with the matrix $E_{N}$, and the discrete version of the theorem of Burchnall and Chaundy on commuting differential operators $[\mathbf{1 6}]$ says there is a polynomial $F(X, Y) \in \mathbb{C}[X, Y]$ of degree $2 N$ in $X$ and degree $2 m$ in $Y$ such that $F\left(A, E_{N}\right)=0$. This result is given in [21] without proof. The main result of this section is to make explicit the polynomial $F$ when $m=1$ and $A$ is symmetric. We suppose in the following that $A$ is a Jacobi matrix with period $N$, so it is tridiagonal and $N$-periodic.

Theorem 2.1. Let $A$ be a Jacobi matrix, which is symmetric and $N$-periodic, then there is a polynomial $\tilde{P}(X)$ in $\mathbb{C}[X]$ of degree $N$, such that $\tilde{P}(A)=E_{N}$. Furthermore, the spectrum $\sigma(A)$ of $A$ is compact in $\mathbb{C}$ and the polynomial $\tilde{P}(X)$ is, up to a constant factor, the Chebyshev polynomial of degree $N$ of $\sigma(A)$.

Proof. To simplify the notation, we write the operator $A$ as

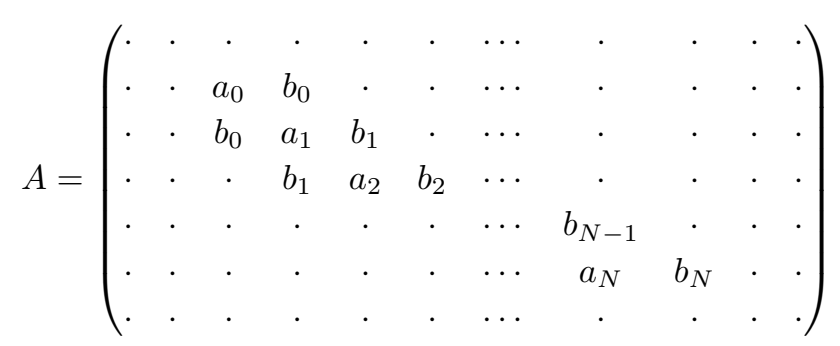

with $a_{i+N}=a_{i}, b_{i+N}=b_{i}, i \in \mathbb{Z}$. From the commutation relation $A E_{N}=E_{N} A$ and from the fact that the eigenspaces of these two operators are finite dimensional, a common eigenvector of $A$ and $E_{N}, y=\left(y_{k}\right)_{k \in \mathbb{Z}}$, exists. We denote by $h$ and $\mu$ the corresponding 
eigenvalues. Hence there exist relations of the form

$$
\begin{aligned}
b_{0} y_{0}+a_{1} y_{1}+b_{1} y_{2} & =h y_{1}, \\
b_{1} y_{1}+a_{2} y_{2}+b_{2} y_{3} & =h y_{2}, \\
& \vdots \\
b_{N-1} y_{N-1}+a_{N} y_{N}+b_{N} y_{N+1} & =h y_{N}, \\
b_{0} y_{N}+a_{1} y_{N+1}+b_{1} y_{N+2} & =h y_{N+1}, \\
b_{1} y_{N+1}+a_{2} y_{N+2}+b_{2} y_{N+3} & =h y_{N+2}, \\
& \vdots \\
b_{N-1} y_{2 N-1}+a_{N} y_{2 N}+b_{N} y_{2 N+1} & =h y_{2 N}, \\
y_{0}+y_{2 N} & =\mu y_{N}, \\
y_{1}+y_{2 N+1} & =\mu y_{N+1} .
\end{aligned}
$$

Because these equations must be consistent, the eigenvalues $h$ and $\mu$ must satisfy $F(h, \mu)=0$, where $F(h, \mu)$ is the following determinant of order $2 N+2$ :

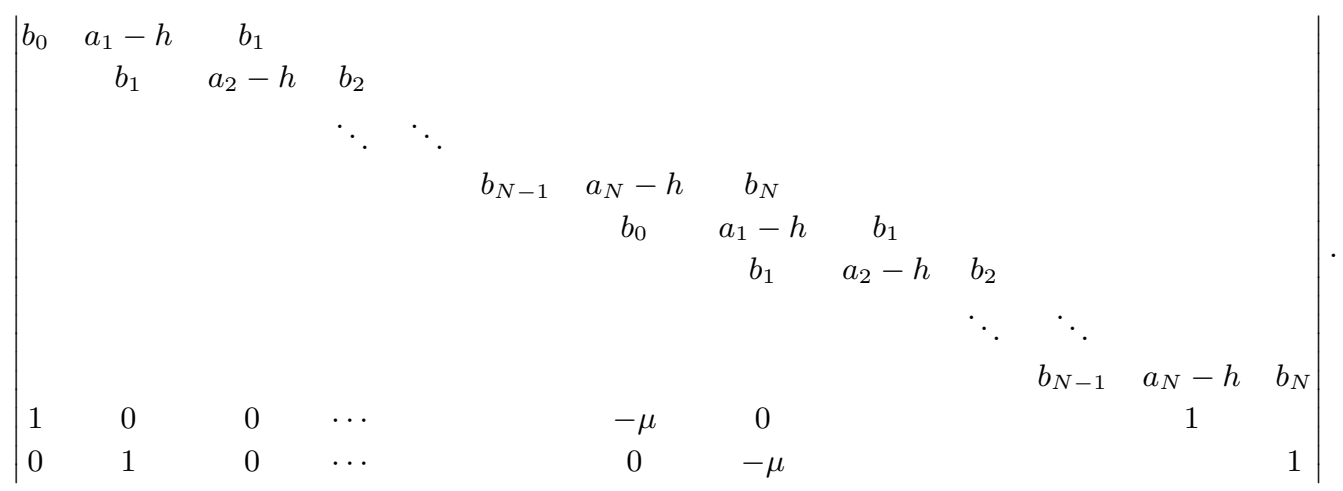

The coefficient $a_{1}-h$ in the centre of the determinant is on the $(N+1)$ th row and on the $(N+1)$ th column. The polynomial $F(h, \mu)$ is of degree $2 N$ in $h$ and of degree 2 in $\mu$. We would like to compute this polynomial explicitly in order to show that it is a perfect square in $\mathbb{C}[h, \mu]$. First of all, $F(h, \mu)$ can be reduced to the determinant of order $2 N$ :

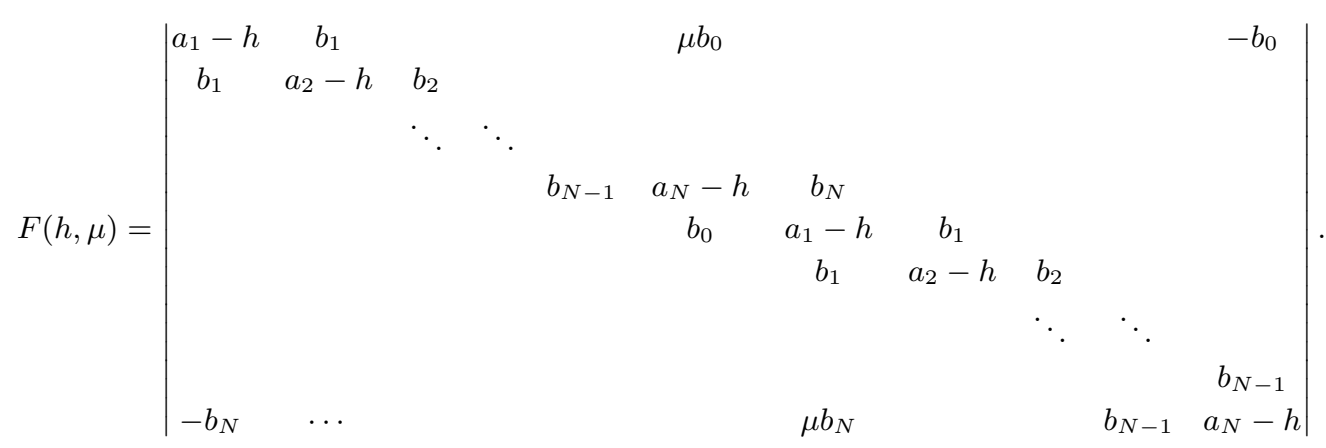


And if

$$
\Delta(i, j)=\left|\begin{array}{cccccc}
a_{i}-h & b_{i} & \ldots & \ldots & \ldots & \ldots \\
b_{i} & a_{i+1}-h & b_{i+1} & \ldots & \ldots & \ldots \\
\cdots & \ldots & \ldots & \ldots & \ldots & \ldots \\
\cdots & \ldots & \ldots & \ldots & \ldots & \ldots \\
\cdots & \ldots & \ldots & b_{j-2} & a_{j-1}-h & b_{j-1} \\
\cdots & \ldots & \ldots & \cdots & b_{j-1} & a_{j}-h
\end{array}\right|
$$

some long but simple computations yield the following.

Lemma 2.2. If $P(h)=\Delta(2, N)-b_{0}^{2} \Delta(2, N-1)$, the polynomial $F(h, \mu)$ is given by

$$
F(h, \mu)=\left(P(h)+(-1)^{N+1} \mu \prod_{0 \leqslant i \leqslant N-1} b_{i}\right)^{2} .
$$

Consequently, if we denote by $\mathcal{B}$ the product $\prod_{i=0}^{N-1} b_{i}$, the polynomial $\tilde{P}(X)$ in the statement of the theorem is

$$
\tilde{P}(X)=\frac{(-1)^{N}}{\mathcal{B}} P(X) .
$$

We will refer to $\mathcal{B}=\prod_{i=0}^{N-1} b_{i}$ as the modulus of the Jacobi matrix $A$ and to $P(X)$ as the Narman polynomial. From the relation $\tilde{P}(A)=E_{N}$ and the fact that the spectrum $\sigma\left(E_{N}\right)$ of $E_{N}$ is the interval $[-2,2]$, we obtain from the functional calculus that the spectrum $\sigma(A)$ of $A$ is the inverse image of $[-2,2]$ under $\tilde{P}$. It is a compact set in $\mathbb{C}$ consisting of arcs which may have common endpoints [21, Theorem 3]. According to a transfer theorem of Fekete [12], we have that

$$
\mathcal{C}(\sigma(A))=|\mathcal{B}|^{1 / N}
$$

We now turn to the last statement of our theorem. We use a result of Ostrovskii, Pakovitch and Zaidenberg [22]; we first recall some definitions. Let $D=D(a, r)$ be a closed disc centred at $a \in \mathbb{C}$ and of radius $r$. We say that a compact $K \subset D \operatorname{supports} D$ if $D$ is the (unique) disc of smallest radius which contains $K$. For a given compact $K \subset \mathbb{C}$, a monic polynomial $p(z) \in \mathbb{C}[z]$ of degree $n \geqslant 0$ is called the $n$th polynomial of least deviation (from zero) or the Chebyshev polynomial of degree $n$ if $\|p\|_{K} \leqslant\|q\|_{K}$ for any monic polynomial $q(z) \in \mathbb{C}[z]$ of degree $n$, where $\|p\|_{K}=\max _{z \in K}\{|p(z)|\}$. The classical inequality of Jung asserts that each compact convex $K$ of diameter $\delta$ is contained in a closed disc $D(a, \rho)$, with $\frac{1}{2} \delta \leqslant \rho \leqslant \delta$. The following theorem is the main result in [22].

Theorem 2.3. Let $\Delta_{r}=\Delta(0, r) \subset \mathbb{C}$ be the disc of radius $r$ centred at the origin, $K \subset \Delta_{r}$ be a supporting compact of $\Delta_{r}$, and $p \in \mathbb{C}[z]$ be a monic polynomial of degree $n$. Then $p$ is the unique $n$th polynomial of least deviation on $K_{p}=p^{-1}(K)$.

This theorem shows clearly that the polynomial $(-1)^{N} P(X)$ is the Chebyshev polynomial of $E=\sigma=\sigma(T)$, the diameter $\left[-|\mathcal{B}|^{1 / N},|\mathcal{B}|^{1 / N}\right]$ supporting the disc centred at the origin, with radius $|\mathcal{B}|^{1 / N}$. 
We have thus established, under the preceding conditions, a relation between the theorem of Burchnall and Chaundy and the problem of the explicit determination of a Chebyshev polynomial of some degree and the capacity of some compact sets.

It is perhaps worthwhile noticing that Chebyshev polynomials appear in a different kind of commutation problem also related to discrete integrable systems. This is the problem of Fatou and Julia on commuting polynomials $[\mathbf{3 1}, \mathbf{3 6}]$. There is another analysis in [34] in their study of difference operators and the associated algebraic curves. It is possible to compare this analysis with ours by using another interesting result due to Pakovitch [23], also stated in [22]. We recall that the essential feature of Floquet theory for periodic difference operators (as for differential operators) is to look for solutions that are eigenfunctions of the translation (by the period) operator. We use the notation of $[\mathbf{3 3}, \S 1]$ and $[\mathbf{3 2}$, Chapter 7].

Now a given Jacobi matrix $A$ defines a difference operator $T$ of period $N$ with $a, b \in$ $l_{\mathbb{R}}^{\infty}(\mathbb{Z}), a_{n} \neq 0$, and $a_{n+N}=a_{n}, b_{n+N}=b_{n}, n \in \mathbb{Z}$. We put

$$
\mathcal{B}=\prod_{n=0}^{N-1} b_{n}=\prod_{n=0}^{N-1} b_{n+n_{0}}, \quad \mathcal{S}=\sum_{n=0}^{N-1} a_{n}=\sum_{n=0}^{N-1} a_{n+n_{0}}, \quad n_{0} \in \mathbb{Z},
$$

and we consider the solutions of the eigenvalue problem

$$
T \psi(z, n)=z \psi(z, n) .
$$

The operator $T$ is given in terms of the shift operator $D$, and for convenience with a change in sign of the main diagonal, by $T=b D^{+}+b^{-} D^{-}-a, b_{n}^{-}=b_{n-1}, z$ denotes the eigenvalue.

As usual, let $c\left(z, n, n_{0}\right), s\left(z, n, n_{0}\right), z \in \mathbb{C}$, be a fundamental system of solutions to (2.1) satisfying

$$
\begin{aligned}
& c\left(z, n_{0}, n_{0}\right)=s\left(z, n_{0}+1, n_{0}\right)=1, \\
& c\left(z, n_{0}+1, n_{0}\right)=s\left(z, n_{0}, n_{0}\right)=0 .
\end{aligned}
$$

The fundamental matrix is

$$
\begin{aligned}
\phi\left(z, n, n_{0}\right) & =\left(\begin{array}{cc}
c\left(z, n, n_{0}\right) & s\left(z, n, n_{0}\right) \\
c\left(z, n+1, n_{0}\right) & s\left(z, n+1, n_{0}\right)
\end{array}\right) \\
& = \begin{cases}U_{n}(z) \cdots U_{n_{0}+1}(z), & n \geqslant n_{0}+1, \\
1, & n=n_{0}, \\
U_{n+1}^{-1}(z) \cdots U_{n_{0}}^{-1}(z), & n \leqslant n_{0}-1,\end{cases}
\end{aligned}
$$

where

$$
\begin{aligned}
U_{m}(z) & =\frac{1}{b_{m}}\left(\begin{array}{cc}
0 & b_{m} \\
-b_{m-1} & z+a_{m}
\end{array}\right), \\
U_{m}(z)^{-1} & =\frac{1}{b_{m-1}}\left(\begin{array}{cc}
z+a_{m} & -b_{m} \\
b_{m-1} & 0
\end{array}\right) .
\end{aligned}
$$


The Wronskian of two solutions $f, g$ is defined by $W(f, g)_{n}=b_{n}\left(f_{n} g_{n+1}-f_{n+1} g_{n}\right)$. Thus

$$
W\left(c\left(z, ., n_{0}\right), s\left(z, ., n_{0}\right)\right)=b_{n_{0}} .
$$

The most general solution $\psi(z)$ of $(2.1)$ is

$$
\psi(z, n)=\psi\left(z, n_{0}\right) c\left(z, n, n_{0}\right)+\psi\left(z, n_{0}+1\right) s\left(z, n, n_{0}\right)
$$

or

$$
\left(\begin{array}{c}
\psi(z, n) \\
\psi(z, n+1)
\end{array}\right)=\phi\left(z, n, n_{0}\right)\left(\begin{array}{c}
\psi\left(z, n_{0}\right) \\
\psi\left(z, n_{0}+1\right)
\end{array}\right) .
$$

Furthermore,

$$
\begin{aligned}
\operatorname{det}\left(\phi\left(z, n, n_{0}\right)\right) & =\frac{b_{n_{0}}}{b_{n}} \\
\phi\left(z, n, n_{0}\right) & =\phi\left(z, n, n_{1}\right) \phi\left(z, n_{1}, n_{0}\right), \\
\phi\left(z, n, n_{0}\right)^{-1} & =\phi\left(z, n_{0}, n\right) .
\end{aligned}
$$

The monodromy matrix $M(z, n)$ is defined by the fundamental matrix

$$
M(z, n)=\phi(z, n+N, n) .
$$

It follows that $M(z, n)=\phi\left(z, n, n_{0}\right) M\left(z, n_{0}\right) \phi\left(z, n, n_{0}\right)^{-1}$ and $\operatorname{det}(M(z, n))=1$. Finally, the Floquet discriminant $\Delta(z)$ is given by

$$
\Delta(z)=\frac{1}{2} \operatorname{tr}(M(z, n)) .
$$

It is independent of $n$. Similarly, the Floquet multipliers $m_{ \pm}(z)$ are the eigenvalues of $M(z, n)$. They are given by $m_{ \pm}(z)=\Delta(z) \pm\left[\Delta(z)^{2}-1\right]^{1 / 2}$ and are independent of $n$. They verify

$$
m_{+}(z) m_{-}(z)=1, \quad m_{+}(z)+m_{-}(z)=2 \Delta(z) .
$$

Following the presentation of [34], the periodicity of the Jacobi matrix $A$ is expressible by the commutation relation $A D^{N}=D^{N} A$ and the eigenvalues $z$ and $h$ associated with a common eigenvector $f$ are elements of the curve

$$
\begin{aligned}
\mathcal{R}_{0} & =\left\{(z, h) \in \mathbb{C} \times \mathbb{C}^{*} \mid L f=z f, D^{N} f=h f, f \neq 0\right\} \\
& =\left\{(z, h) \in \mathbb{C} \times \mathbb{C}^{*} \mid \operatorname{det}\left(C_{h}-z I\right)=F\left(h, h^{-1}, z\right)=0\right\},
\end{aligned}
$$

where

$$
C_{h}-z I=\left(\begin{array}{cccccc}
a_{1}-z & b_{1} & \cdot & \cdots & . & b_{0} h^{-1} \\
b_{1} & a_{2}-z & b_{2} & \cdots & \cdot & \cdot \\
\cdot & \cdot & \cdot & \cdots & \cdot & b_{N-1} \\
b_{N} h & \cdot & \cdot & \cdots & b_{N-1} & a_{N}-z
\end{array}\right)
$$

It is easy to see that

$$
F\left(h, h^{-1}, z\right)=(-1)^{N+1}\left\{\prod_{i=1}^{N} b_{i}\left(h+h^{-1}\right)-R(z)\right\},
$$


where $R(z)$ is a polynomial of degree $N$. We also have

$$
\mathcal{R}_{0}=\left\{(z, h) \in \mathbb{C} \times \mathbb{C}^{*} \mid\left(h+h^{-1}\right)-2 \Delta(z)=0\right\} .
$$

An important remark can be made at this point: if $V_{z}=\{f, T f=z f\}$, then $V_{z}$ is a vector space over $\mathbb{C}$ of dimension two on which $D^{N}$ acts, and $\Delta(z)=\frac{1}{2} \operatorname{tr} D_{\mid V_{z}}^{N}$ is just the monodromy matrix. From the commutation relation $D^{N} f=h f$ we get $D^{-N} f=h^{-1} f$ and then $\mathcal{R}_{0} \subset \tilde{\mathcal{R}}_{0}$, where

$$
\tilde{\mathcal{R}}_{0}=\left\{(z, h) \in \mathbb{C} \times \mathbb{C}^{*}, L f=z f, E_{N} f=\left(h+h^{-1}\right) f, f \neq 0\right\} .
$$

This means that for each $(z, h) \in \mathcal{R}_{0}, P(z)=h+h^{-1}$ or for each $z$ such that $(z, h) \in \mathcal{R}_{0}$, we have $P(z)=2 \Delta(z)$. From [33, p. 48] we know that the $2 N$ eigenvalues of

$$
Q=\left(\begin{array}{cccccccc}
a_{1} & b_{1} & \cdots & \cdots & \cdots & \cdots & \cdots & a_{N} \\
b_{1} & a_{2} & \cdots & & & & & \\
& \cdots & \cdots & b_{N-1} & & & & \\
& & b_{N-1} & a_{N} & b_{N} & & & \\
& & & b_{N} & a_{1} & b_{1} & & \\
& & & & b_{1} & \cdots & \cdots & \\
a_{N} & \cdots & \cdots & \cdots & \cdots & \cdots & a_{N-1} & a_{N}
\end{array}\right)
$$

are such $z$. Thus we have proved the following proposition.

Proposition 2.4. The Nauman polynomial and the Floquet discriminant are related by $\tilde{P}(z)=2 \Delta(z)$. Consequently, the Riemann surfaces $\tilde{\mathcal{R}}_{0}, \mathcal{R}_{0}$ are identical.

We wish to point out that the result of the preceding proposition can also be obtained by using another interesting theorem of $[\mathbf{2 2}]$, which we cite for completeness.

Theorem 2.5. Let $K \subset \mathbb{C}$ be a compact set that contains at least two points. Suppose that $p, q \in \mathbb{C}[z]$ are two polynomials of the same degree $n$ such that $p^{-1}(K)=q^{-1}(K)$. Then $p=\alpha(q)$, where $\alpha(z)$ is a rotation of $\mathbb{C}$ which preserves $K$.

In fact, the polynomial $T(z)=2 \mathcal{B} \Delta(z)$ is the Chebyshev polynomial of degree $N$ of $\sigma(A)$, with $T^{-1}([-2|\mathcal{B}|, 2|\mathcal{B}|])=\sigma(A)$. But if $\tilde{T}=\tilde{P}, P$ is the Naŭman polynomial, then $\tilde{T}^{-1}([-2|\mathcal{B}|, 2|\mathcal{B}|])=\sigma(A)$. By the preceding theorem, we get again $\tilde{P}(z)=2 \Delta(z)$.

\subsection{Metric properties of polynomials}

In this section, we wish to discuss some metric properties of the spectrum $\sigma(A)$ of a Jacobi matrix $A$ that can be derived directly from results of the preceding section. Our first observation is that the polynomial $F(h, \mu)$ is a determinant, and, for a fixed $\mu$ in $[-2,2], h$ appears as a zero of a relatively simple determinant $\Delta$. We recall a classical result of Hirsch [14] and Bendixon [3]. If $s$ is an eigenvalue of $\left(a_{\lambda, \nu}\right)_{0 \leqslant \lambda, \nu \leqslant n}$ and if $a$ 
is the maximum of $\left|a_{\lambda, \mu}\right|, b$ is the maximum of $\frac{1}{2}\left|a_{\lambda, \nu}+\bar{a}_{\nu, \lambda}\right|$ and $c$ is the maximum of $\frac{1}{2}\left|a_{\lambda, \nu}-\bar{a}_{\nu, \lambda}\right|$, then

$$
|s| \leqslant n a, \quad|\operatorname{Re}(s)| \leqslant n b, \quad|\operatorname{Im}(s)| \leqslant n c .
$$

We return to the determinant $\Delta$, of order $2 N$, and we denote by

$$
\begin{aligned}
& \tilde{a}=\max \left(\left|a_{\nu}\right|,\left|b_{\nu}\right|,|\mu|\left|b_{0}\right|, 0 \leqslant \nu \leqslant N-1\right), \\
& \tilde{b}=\max \left(\left|\operatorname{Re} a_{\nu}\right|,\left|\operatorname{Re} b_{\nu}\right|, \frac{1}{2}|\mu|\left|b_{0}\right|, 0 \leqslant \nu \leqslant N-1\right), \\
& \tilde{c}=\max \left(\left|\operatorname{Im} a_{\nu}\right|,\left|\operatorname{Im} b_{\nu}\right|, \frac{1}{2}|\mu|\left|b_{0}\right|, \quad 0 \leqslant \nu \leqslant N-1\right) .
\end{aligned}
$$

Proposition 2.6. Let $A$ be a Jacobi matrix with complex coefficients, symmetric and of period $N$. The elements $h$ of the spectrum $\sigma(A)$ are such that

(1) $|h| \leqslant 2 N \tilde{a}$,

(2) $\operatorname{Re}(h) \leqslant 2 N \tilde{b}$,

(3) $\operatorname{Im}(h) \leqslant 2 N \tilde{c}$.

In particular, the arclength $\mathcal{L}(A)$ of the convex hull of $\sigma(A)$ satisfies

$$
\mathcal{L}(A) \leqslant 2 \pi \max \left(\left|a_{\nu}\right|,\left|b_{\nu}\right|, 0 \leqslant \nu \leqslant N-1 ; 2\left|b_{0}\right|\right) .
$$

There is a completely different approach to this question leading to another kind of result. Cartan's Lemma [4] says that if $p(z)$ is a monic polynomial of degree $N$,

$$
p(z)=\prod_{1 \leqslant i \leqslant N}\left(z-\alpha_{i}\right),
$$

then the inequality $|p(z)|>M$ holds for $z$ outside discs, the sum of their radii being at most $2 \mathrm{e} M^{1 / N}$. There is also a formulation in terms of Hausdorff measures, which we formulate in the following way [9]: for positive $\epsilon$ and $\alpha$, the set $E(p, \epsilon)=\{z \in \mathbb{C}|| p(z) \mid \leqslant$ $\left.\epsilon^{N}\right\}$ can be covered by at most $N$ discs $D_{j}$ such that the diameters $d\left(D_{j}\right)$ satisfy

$$
\sum_{1 \leqslant j \leqslant l} d\left(D_{j}\right)^{\alpha} \leqslant \mathrm{e} 4^{\alpha} \epsilon^{\alpha}
$$

The constant $\mathrm{e} 4^{\alpha}$ is not sharp and it is conjectured that if $\alpha=1$, the best constant is 4 instead of 4e. The spectrum $\sigma(A)$ is mapped into the closed disc $\{|w| \leqslant 2|B|\}$. These facts applied to the Năman polynomial with $\epsilon^{N}=2|B|$ and Theorem 3 of [21] lead to the following result.

Theorem 2.7. Let $A$ be a Jacobi matrix, real, symmetric and $N$-periodic with modulus $\mathcal{B}=\prod_{i=0}^{N-1} b_{i}$. Let $I_{i}=\left[E_{2 i-2}, E_{2 i-1}\right], i=1, \ldots, N$, be the components of the spectrum $\sigma(A)$ and let $l_{i}$ be the length of $I_{i}$. Then for each positive $\alpha$,

(1) $\sum_{1 \leqslant j \leqslant N} l_{j}^{\alpha} \leqslant \mathrm{e} 4^{\alpha}(2|B|)^{\alpha / N}$,

(2) $\mathcal{C}(\sigma(A)) \leqslant \frac{1}{4} \sum_{1 \leqslant j \leqslant N} l_{j} \leqslant \mathrm{e}(2|B|)^{1 / N}$. 
It follows from this result, from the equality $\mathcal{C}(\sigma(A))=|B|^{1 / N}$ and the subadditivity of the capacity that the following inequalities hold:

$$
\mathcal{C}(\sigma(A)) \leqslant \sum_{1 \leqslant i \leqslant N} \mathcal{C}\left(I_{i}\right) \leqslant \mathrm{e} 2^{1 / N} \mathcal{C}(\sigma(A))
$$

or

$$
\mathcal{C}(\sigma(A)) \leqslant \frac{1}{4} \sum_{1 \leqslant i \leqslant N}\left(E_{2 i-1}-E_{2 i-2}\right) \leqslant \mathrm{e} 2^{1 / N} \mathcal{C}(\sigma(A))
$$

Another kind of result is given by the next theorem.

Theorem 2.8. Let $\sigma=\sigma(A)$ be the spectrum of a real, symmetric and $N$-periodic Jacobi matrix $A$ with modulus $\mathcal{B}$. The arclength $\mathcal{L}(A)$ of the convex hull of $\sigma(A)$ satisfies

$$
\mathcal{L}(A) \leqslant \pi(\sqrt{10}-3 \sqrt{2}+4) N|B|^{1 / N} \leqslant 9,173 N|B|^{1 / N} .
$$

Proof. We adapt ideas of Pommerenke [28] and Eremenko and Hayman [10]. The main goal here is to see (as we will do in several places in this paper) that methods of conformal mapping theory can be used in problems on Jacobi matrices. We begin with a lemma.

Lemma 2.9. For a connected compact set $K$ in $\mathbb{C}$ of capacity $\mathcal{C}(K)$, the perimeter $\mathcal{L}_{0}$ of the convex hull of $K$ is at most $\pi(\sqrt{10}-3 \sqrt{2}+4) \mathcal{C}(K)$.

To prove this lemma, we proceed as in $[\mathbf{2 8}$, Satz 5]. We consider the map $f$ sending $\{|z|>\mathcal{C}(K)\}$ onto the complement of $K$ in the Riemann sphere $\hat{\mathbb{C}}$. The map $g(u)=$ $f(\mathcal{C}(K) u)$ has the expansion

$$
g(u)=\mathcal{C}(K) u+a_{0}+\frac{a_{1}}{u}+\cdots
$$

and $g$ maps $\{|u|>1\}$ onto the complement of $K$. We apply Pommerenke's methods [28, Hillfssatz 3, p. 63] to $\tilde{f}, \tilde{f}(u)=\mathcal{C}(K)^{-1} g(u)$. Let $c \in K$ be a given point. Then the function $\phi$ defined by

$$
\phi(\xi)=\left(\tilde{f}\left(\xi^{-1}\right)-\frac{c}{\mathcal{C}(K)}\right)^{-1}=\xi+\cdots
$$

is one to one in the unit disc $\{|\xi|<1\}$ and satisfies $|\phi(\xi)| \leqslant\left(|\xi| /(1-|\xi|)^{2}\right)$. This implies that $\mathcal{C}(K)(|z|-2+(1 /|z|)) \leqslant|f(\mathcal{C}(K) z)-c|$, and, if $r \geqslant \mathcal{C}(K)$ and $\mathcal{L}(r)=$ $r \int_{0}^{2 \pi} f^{\prime}\left(r \mathrm{e}^{2 \mathrm{i} \pi \theta}\right) \mathrm{d} \theta$ is the length of the image of the circle $\{|z|=r\}$ under $f, \mathcal{L}_{0} \leqslant \mathcal{L}(r)-$ $2 \pi r(r-2+(1 / r))$. Now as in Pommerenke's arguments [28, pp. 68, 69], we write $f(z)=$ $z+\sum_{0}^{\infty} a_{n} z^{-n}$ and we have

$$
f(\mathcal{C}(K) u)=g(u)=\mathcal{C}(K) u+\sum_{0}^{\infty} a_{n} \mathcal{C}(K)^{-n} u^{-n}
$$


defined for $|u|>1$. It follows that if $R>1$ is given and $r=R \mathcal{C}(K)$, then

$$
\mathcal{L}(r) \leqslant 2 \pi \mathcal{C}(K)\left(R^{2}+\sum_{1}^{\infty} n\left|a_{n}\right|^{2} n \mathcal{C}(K)^{-2 n} R^{-2 n}\right)^{1 / 2} .
$$

For $R=\sqrt{2}$ or $r=\sqrt{2} \mathcal{C}(K)$, we get

$$
\mathcal{L}_{0} \leqslant\left(\pi \sqrt{10}-2 \pi\left(\sqrt{2}-2+\frac{1}{2} \sqrt{2}\right)\right) \mathcal{C}(K)
$$

This proves the lemma.

To complete the proof of the theorem, we consider a general monic polynomial $p(z)$ of degree $d$, we denote by $E(p, c)$ the lemniscate $E(p, c)=\{z \in \mathbb{C}|| p(z) \mid=c\}$ and we denote by $|E(p, c)|$ its arclength. Here we adapt an argument of Eremenko and Hayman [10]. If $d$ is a given positive integer, we call an extremal polynomial $p^{*}$ any polynomial that maximizes $|E(p, c)|$ in the set of all monic polynomials of degree $d$. For such a polynomial $p^{*}$, the lemniscate $E\left(p^{*}, c\right)=\left\{z \in \mathbb{C}|| p^{*}(z) \mid=c\right\}$ is a connected set. By the transfer theorem of Fekete used earlier, the capacity $\mathcal{C}(E(p, c))$ is $c^{1 / d}$. By the preceding lemma, we conclude that the arclength $\widetilde{\mathcal{E}(p, c)}$ of the convex hull $\widetilde{E(p, c)}$ of $E(p, c)$ is at most $\pi(\sqrt{10}-3 \sqrt{2}+4) c^{1 / d}$. For each real $x$, let $N_{E}(\theta, x)$ be the number of common points of $E(p, x)$ with the line $\left\{z \in \mathbb{C}, \operatorname{Re}\left(z \mathrm{e}^{-\mathrm{i} \theta}\right)=x\right\}$. The integral-geometric formula (see [5] and $[\mathbf{1 0}$, p. 144]) gives the length of the lemniscate $E(p, c)$ as

$$
|E(p, c)|=|\{z \in \mathbb{C}|| p(z) \mid=c\}|=\frac{1}{2} \int_{0}^{\pi} \int_{-\infty}^{\infty} N_{E(p, c)}(\theta, x) \mathrm{d} x \mathrm{~d} \theta .
$$

Now it is clear that if $N_{\overparen{E(\theta, x)}}$ is the number of common points of $\widetilde{E(p, c)}$ with the line $\left\{z \in \mathbb{C}, \operatorname{Re}\left(z \mathrm{e}^{-\mathrm{i} \theta}\right)=x\right\}$, then $d N_{E}(\theta, x) \leqslant N_{\widetilde{E(\theta, x)}}$ and so

$$
|E(p, c)| \leqslant d|\widetilde{E(p, c)}| \leqslant d c^{1 / d} \pi(\sqrt{10}-3 \sqrt{2}+4)<9.173 d c^{1 / d} .
$$

The proof of the theorem is complete.

It seems that the results of this theorem are of different character to those of the preceding proposition.

\subsection{Differential equations and oscillations}

In the present section, we wish to provide three approaches for obtaining a Chebyshev polynomial of some degree of a finite union of closed intervals. In [23], Pakovitch defines the notion of an elliptic polynomial and proves that, up a multiplicative factor, an elliptic polynomial is the Chebyshev polynomial of a union of two intervals. For a similar presentation and a modular interpretation see Hirzebruch, Berger and Jung [15, pp. 107, 187]. In some sense, this is a genus-one situation. We will extend this notion to the hyperelliptic case and this will be our first approach. The second approach will show how oscillation properties of a polynomial can make it a Chebyshev polynomial. Finally, the third approach will explain the differential equation character of being a Chebyshev polynomial. We start by an illustrating connection. 
Theorem A. Let $E=\left[E_{1}, E_{2}\right] \cup\left[E_{3}, E_{4}\right] \cup \cdots \cup\left[E_{2 g+1}, E_{2 g+2}\right]$ be a finite union of closed intervals, there exists a Jacobi matrix $A$, real, symmetric and $N$-periodic, $g+1 \leqslant N$, with the spectrum $\sigma(A)$ equal to $E$ if and only if the $N$ th Chebyshev polynomial of $E$ oscillates $N$ times over $E$.

We recall that a polynomial $P(x)$ with real coefficients oscillates $N$ times between $\pm M$ on an interval $E^{\prime}$ if $|P(x)|=M$ has $N+1$ solutions on $E^{\prime}$ with alternating signs. The proof of Theorem A becomes apparent after the introduction of the following notion, which extends the definition of elliptic polynomials of $[\mathbf{2 3}]$.

Definition 2.10. A real polynomial $P$ of degree $n$ is called $g$-hyperelliptic if and only if it satisfies a differential equation of the form

$$
P^{2}(z)-\left[\frac{P^{\prime}(z)}{n\left(z-x_{1}\right) \cdots\left(z-x_{g}\right)}\right]^{2} R_{2 g+2}(z)=1,
$$

with $x_{1}, \ldots, x_{g} \in \mathbb{C}$, and if $R$ is a polynomial of degree $2 g+2$ with simple and real roots.

From (2.3), we see that $x_{1}, \ldots, x_{g}$ are zeros of the derivative $P^{\prime}$. There is a deep link between the extremal properties of the Chebyshev polynomial and their oscillations properties. In the case of one interval, this is contained in the Equal Ripple Theorem, essentially due to Chebyshev, which concerns the quantity

$$
\mu=\min _{\{p\}} \max _{-1 \leqslant x \leqslant 1}|f(x)-p(x)|,
$$

where $f$ is continuous on the interval $[-1,1]$ and $p(x)$ is an arbitrary polynomial of degree at most $N$.

Theorem 2.11 (the Equal Ripple Theorem [8]). There is a unique polynomial of best approximation to $f(x)$ in $[-1,1]$ and it is characterized by the existence of a sequence of at least $N+2$ points at which the error function $e(x)=f(x)-p(x)$ assumes the values $\pm \mu$, the signs alternating.

If $f(x)=x^{N}$ is approximated by polynomials of degree less than or equal to $N-1$, we obtain the $N$ th Chebyshev polynomial $T_{N}(X)$ or the Zolotarev polynomial. This equioscillation property, combined with the fact that all critical values are equal up to signs, is converted into a differential equation. We wish to explain briefly what happens for several intervals. We refer to $[\mathbf{2 9}]$ and $[\mathbf{3 0}]$ for the details. If $E=\left[E_{1}, E_{2}\right] \cup\left[E_{3}, E_{4}\right] \cup$ $\cdots \cup\left[E_{2 g+1}, E_{2 g+2}\right]$, we can no longer say that the Chebyshev polynomial of degree $N$ oscillates $N$ times on $E$ between $\pm M$, but we can find $N+1$ special points of $E$ where $T_{N}(X)$ takes the values $\pm M$ with alternating signs. If two consecutive points of this special set are contained in the same component of $E$, then $T_{N}(X)$ oscillates from $+M$ to $-M$ or from $-M$ to $+M$, but if they are in different components, then various behaviours are possible on the finite gaps (bounded components of $\mathbb{R} \backslash E$ ). If we require that in $\mathbb{R} \backslash E,\left|T_{N}(x)\right|>M$, then $T_{N}(X)$ will be the $N$ th Chebyshev polynomial of $E$ because it oscillates $N$ times between $\pm M$. These remarks motivate Equation (2.3). In fact the roots of $T_{N}(X)= \pm M$ are all double roots, except for the ends $E_{i}, 1 \leqslant i \leqslant 2 g+2$. 
There are $N-g-1$ of these double roots which are also roots of $T_{N}^{\prime}(X)$ and this last equation has $g$ additional roots $c_{1}, c_{2}, \ldots, c_{g}$ localized in the gaps, so that

$$
E_{1}<E_{2}<c_{1}<E_{3}<E_{4}<c_{2}<\cdots<c_{g}<E_{2 g+1}<E_{2 g+2} .
$$

This leads to the identity

$$
\frac{N^{2}\left[T_{N}(x)^{2}-M^{2}\right]}{\left(x-E_{1}\right)\left(x-E_{2}\right) \cdots\left(x-E_{2 g+1}\right)\left(x-E_{2 g+2}\right)}=\frac{T_{N}^{\prime}(x)^{2}}{\left(x-c_{1}\right)^{2}\left(x-c_{2}\right)^{2} \cdots\left(x-c_{g}\right)^{2}} .
$$

This is Equation (2.3), a nonlinear differential equation of first order, which admits the solution

$$
f(z)=M \cosh \left(N \int_{E_{2 g+2}}^{z} \sqrt{Q(t)} \mathrm{d} t\right)
$$

where for $E_{2 g+2} \leqslant z=x \in \mathbb{R}$, the positive square root is used and

$$
Q(t)=\frac{\left(t-c_{1}\right)^{2}\left(t-c_{2}\right)^{2} \cdots\left(t-c_{g}\right)^{2}}{\left(t-E_{1}\right)\left(t-E_{2}\right) \cdots\left(t-E_{2 g+1}\right)\left(t-E_{2 g+2}\right)} .
$$

Now we are able to formulate the important result of Robinson $[\mathbf{2 9}, \mathbf{3 0}]$.

Theorem 2.12. In order that the function $f(z)$ given by (2.4) should be single valued, it is necessary that

$$
\int_{E_{2 k}}^{E_{2 k+1}} \sqrt{Q(t)} \mathrm{d} t=0, \quad k=1,2, \ldots, g .
$$

Further, in order that $f(z)$ oscillates $n_{k}$ times between $\pm M$ in the $k$ th interval, it must satisfy

$$
\int_{E_{2 k-1}}^{E_{2 k}} \sqrt{Q(t)} \mathrm{d} t= \pm \frac{n_{k} \pi \mathrm{i}}{N}, \quad k=1,2, \ldots, g+1, \quad n_{1}+n_{2}+\cdots+n_{g+1}=N .
$$

If these conditions are satisfied, then the function $f(z)=T_{N}(z)$ is the $N$ th Chebyshev polynomial of $E=\left[E_{1}, E_{2}\right] \cup\left[E_{3}, E_{4}\right] \cup \cdots \cup\left[E_{2 g+1}, E_{2 g+2}\right]$.

The origin of the present work was the desire to understand the connection between the works of Robinson [29] and Krichever [18]. It is remarkable that Theorem 2.12 and Definition 2.10 appeared recently and in another context in Peherstorfer [24-27]. We now give another formulation of Theorem A.

Theorem B. Let $P(z)=a_{n} z^{n}+\cdots+a_{1} z+a_{0}$ be a $g$-hyperelliptic real polynomial such that $R_{2 g+2}(z)=\left(z-E_{1}\right)\left(z-E_{2}\right) \cdots\left(z-E_{2 g+2}\right)$. Then the polynomial $\tilde{P}(z)=\left(1 /\left|a_{n}\right|\right) P(z)$ is of least deviation from zero among all the monic polynomials of degree $n$. The modulus of this deviation is $1 /\left|a_{n}\right|$ and $\tilde{P}$ is the Chebyshev polynomial of degree $n$ of $E=\left[E_{1}, E_{2}\right] \cup\left[E_{3}, E_{4}\right] \cup \cdots \cup\left[E_{2 g+1}, E_{2 g+2}\right]$. 
The proofs of Theorem A and Theorem B depend on the crucial observation that the Floquet discriminant $\Delta(z)$ given by $(2.2)$ of a real $N$-periodic Jacobi matrix is an $N$-hyperelliptic real polynomial. More precisely, we denote by $\left\{\tilde{E}_{l}\right\}_{1 \leqslant l \leqslant 2 N}$ the zeros of $\Delta(z)^{2}-1$ and we write

$$
\begin{gathered}
\Delta(z)^{2}-1=\frac{1}{4 A^{2}} \prod_{l=1}^{2 N}\left(z-\tilde{E}_{l}\right), \\
\Delta(z) \mp 1=\frac{1}{2 A} \prod_{j=1}^{N}\left(z-E_{j}^{ \pm}\right) .
\end{gathered}
$$

According to $[\mathbf{6}, \mathbf{3 3}]$, the zeros $\left\{E_{j}^{+}\right\}_{1 \leqslant j \leqslant N}$ and $\left\{E_{j}^{-}\right\}_{1 \leqslant j \leqslant N}$ are, respectively, the eigenvalues of the periodic Jacobi matrix $\tilde{H}_{n_{0}}^{+}$and the anti-periodic Jacobi matrix $\tilde{H}_{n_{0}}^{-}$given by

$$
\tilde{H}_{n_{0}}^{ \pm}=\left(\begin{array}{ccccc}
-a_{n_{0}+1} & b_{n_{0}+1} & & & \pm b_{n_{0}+N} \\
b_{n_{0}+1} & -a_{n_{0}+2} & & & \\
& & \ldots & & \\
& & & -a_{n_{0}+N-1} & b_{n_{0}+N-1} \\
\pm b_{n_{0}+N} & & & b_{n_{0}+N-1} & -a_{n_{0}+N}
\end{array}\right) .
$$

If $N$ is even, we have

$$
E_{1}^{+}<E_{1}^{-} \leqslant E_{2}^{-}<E_{2}^{+} \leqslant E_{3}^{+} \leqslant \cdots \leqslant E_{N-1}^{+}<E_{N-1}^{-} \leqslant E_{N}^{-}<E_{N}^{+},
$$

while if $N$ is odd,

$$
E_{1}^{-}<E_{1}^{+} \leqslant E_{2}^{+}<E_{2}^{-} \leqslant E_{3}^{-} \leqslant \cdots \leqslant E_{N-2}^{-}<E_{N-2}^{+} \leqslant E_{N-1}^{+}<E_{N-1}^{-} \leqslant E_{N}^{-}<E_{N}^{+} .
$$

The set $\left\{\tilde{E}_{l}\right\}_{1 \leqslant l \leqslant 2 N}$ coincides with the corresponding sequence $\left(E_{j}^{ \pm}\right)$. The spectrum $\sigma(H)$ of $H$ is characterized by

$$
\sigma(H)=\{\lambda \in \mathbb{R},|\Delta(\lambda)| \leqslant 1\}=\bigcup_{j=1}^{N}\left[\tilde{E}_{2 j-1}, \tilde{E}_{2 j}\right] .
$$

When the spectral gaps of $H$ are open, we have $g=N-1$,

$$
\left[\prod_{l=1}^{2 N}\left(z-\tilde{E}_{l}\right)\right]^{1 / 2}=R_{2 g+2}^{1 / 2}(z)=2 A\left[\Delta(z)^{2}-1\right]^{1 / 2} .
$$

When the spectral gaps of $H$ are closed, we introduce

$$
J^{\prime}=\left\{1 \leqslant j^{\prime} \leqslant N-1, \tilde{E}_{2 j^{\prime}}=\tilde{E}_{2 j^{\prime}+1}\right\}, \quad J=\{1,2, \ldots, 2 N\} \backslash\left\{j^{\prime}, j^{\prime}+1, j^{\prime} \in J^{\prime}\right\}
$$

and define

$$
Q(z)=\frac{1}{2 A} \prod_{j^{\prime} \in J^{\prime}}\left(z-\tilde{E}_{2 j^{\prime}-1}\right), \quad R_{2 g+2}(z)=\prod_{j \in J}\left(z-\tilde{E}_{j}\right) .
$$


We identify $\left\{\tilde{E}_{j}\right\}_{j \in J}$ and $\left\{E_{m}\right\}_{1 \leqslant m \leqslant 2 g+2}$ and we get the important relation

$$
\left[\Delta(z)^{2}-1\right]^{1 / 2}=R_{2 g+2}^{1 / 2}(z) Q(z),
$$

with $g=N-1-\left|J^{\prime}\right|=N-1-\operatorname{deg}(Q)=(|J|-2) / 2$. The relation (2.8) says that the Floquet discriminant $\Delta(z)$ is a real $N$-hyperelliptic polynomial. One can check that

$$
Q(z)=\frac{\Delta^{\prime}(z)}{N\left(z-x_{1}\right) \cdots\left(z-x_{g}\right)},
$$

where $x_{1}, \ldots, x_{g}$ are zeros of $\Delta^{\prime}(z), x_{i} \in\left[E_{2 i}, E_{2 i+1}\right]$ and the zeros $\tilde{E}_{2 j^{\prime}}, j^{\prime} \in J^{\prime}$, of $Q(z)$ are double roots of $\Delta(z)^{2}=1$. Furthermore, $\operatorname{deg} Q=N-1-g, \operatorname{deg} \Delta^{\prime}=N-1$ and at the relative maxima and minima of $\Delta(z), \Delta^{\prime}(z)=0$. Finally, we have $|\Delta(z)| \geqslant 1$ on the gaps.

\section{Inverse problems}

\subsection{Statements of the inverse problems}

In our formulation of the inverse problem, we closely follow the presentations of [6] and [32], where a complete list of references is given. The principal topic of this section is to associate with a finite union of intervals $E$ a Jacobi matrix $A$ whose spectrum is $E$.

We start with a union of $N=g+1$ intervals $E=\left[E_{1}, E_{2}\right] \cup\left[E_{3}, E_{4}\right] \cup \cdots \cup\left[E_{2 g-1}, E_{2 g}\right] \cup$ $\left[E_{2 g+1}, E_{2 g+2}\right]$ and we consider the hyperelliptic curve $X_{g}$ associated with the equation

$$
y^{2}=R_{2 g+2}(z)=\prod_{m=1}^{2 g+2}\left(z-E_{m}\right) .
$$

There is a natural projection

$$
\begin{gathered}
\tilde{\pi}: X_{g} \rightarrow \hat{\mathbb{C}}, \\
P=\left(z, \pm R_{2 g+2}(z)^{1 / 2}\right) \rightarrow z \\
\infty_{1}, \infty_{2} \rightarrow \infty .
\end{gathered}
$$

For an arbitrary $n_{0}$ in $\mathbb{Z}$, we consider, as in $\left[6\right.$, p. 14], $g$ points in $X_{g}$,

$$
\left\{\hat{\mu}_{j}\left(n_{0}\right)\right\}_{1 \leqslant j \leqslant g} \subset X_{g}, \quad \tilde{\pi}\left(\hat{\mu}_{j}\left(n_{0}\right)\right)=\mu_{j}\left(n_{0}\right) \in\left[E_{2 j}, E_{2 j+1}\right], \quad 1 \leqslant j \leqslant g,
$$

and we introduce the polynomials $F_{g}\left(z, n_{0}\right)=\prod_{j=1}^{g}\left(z-\mu_{j}\left(n_{0}\right)\right)$,

$$
G_{g+1}\left(z, n_{0}\right)=-\sum_{j=1}^{g} R_{2 g+2}^{1 / 2}\left(\hat{\mu}_{j}\left(n_{0}\right)\right) \prod_{k=1, k \neq j}^{g} \frac{z-\mu_{k}\left(n_{0}\right)}{\mu_{j}\left(n_{0}\right)-\mu_{k}\left(n_{0}\right)}-\left(z+a_{n_{0}}\right) F_{g}\left(z, n_{0}\right),
$$

where

$$
a_{n_{0}}=\sum_{j=1}^{g} \mu_{j}\left(n_{0}\right)-\frac{1}{2} \sum_{m=1}^{2 g+2} E_{m}
$$


Next, we define $F_{g}\left(z, n_{0}+1\right)$ by

$$
G_{g+1}\left(z, n_{0}\right)^{2}-4 b_{n_{0}}^{2} F_{g}\left(z, n_{0}\right) F_{g}\left(z, n_{0}+1\right)=R_{2 g+2}(z),
$$

where the constant $b_{n_{0}}^{2} \neq 0$ is such that $F_{g}\left(z, n_{0}+1\right)$ is a monic polynomial in $z$. We observe that

$$
b_{n_{0}}^{2} F_{g}\left(z, n_{0}\right) F_{g}\left(z, n_{0}+1\right) \geqslant 0
$$

for $z=E_{2 j}, E_{2 j+1}, E_{2 g+1}$. The right-hand side of the preceding inequality has two zeros in $\left[E_{2 j-1}, E_{2 j}\right]$. Hence $F_{g}\left(z, n_{0}+1\right)$ is of the form

$$
F_{g}\left(z, n_{0}+1\right)=\prod_{j=1}^{g}\left(z-\mu_{j}\left(n_{0}+1\right)\right), \quad \mu_{j}\left(n_{0}+1\right) \in\left[E_{2 j}, E_{2 j+1}\right], \quad 1 \leqslant j \leqslant g .
$$

By using (3.2) with $z=E_{2 g+1}$, we see that $b_{n_{0}}^{2} \neq 0$ and then $b_{n_{0}}>0$. Furthermore,

$$
b_{n_{0}}^{2}=\frac{1}{2} \sum_{j=1}^{g} R_{2 g+2}^{1 / 2}\left(\hat{\mu}_{j}\left(n_{0}\right)\right) \prod_{k=1, k \neq j}^{g}\left(\mu_{j}\left(n_{0}\right)-\mu_{k}\left(n_{0}\right)\right)^{-1}-\frac{1}{4}\left(a_{n_{0}}^{2}+a_{n_{0}}^{(2)}\right)>0,
$$

where

$$
a_{n_{0}}^{(2)}=\sum_{j=1}^{g} \mu_{j}\left(n_{0}\right)^{2}-\frac{1}{2} \sum_{m=1}^{2 g+2} E_{m}^{2}, \quad k \in \mathbb{N} .
$$

We have thus obtained a set $\left\{\hat{\mu}_{j}\left(n_{0}+1\right)\right\}_{1 \leqslant j \leqslant g}$ from $\left\{\hat{\mu}_{j}\left(n_{0}\right)\right\}_{1 \leqslant j \leqslant g}$. Thus we can define recursively the sets $\left\{\hat{\mu}_{j}(n)\right\}_{1 \leqslant j \leqslant g}$ for all $n \in \mathbb{Z}$, as these calculations can also be made in the opposite direction. Summing up, we have the following.

Lemma 3.1. Given a sequence $\left\{\hat{\mu}_{j}\left(n_{0}\right)\right\}_{1 \leqslant j \leqslant g}$ satisfying (3.1) as initial data, one can define $\left\{\hat{\mu}_{j}(n)\right\}_{1 \leqslant j \leqslant g}$ for every $n \in \mathbb{Z}$ still satisfying (3.1), that is

$$
\tilde{\pi}\left(\hat{\mu}_{j}(n)\right)=\mu_{j}(n) \in\left[E_{2 j}, E_{2 j+1}\right], \quad 1 \leqslant j \leqslant g, \quad n \in \mathbb{Z} .
$$

Moreover, one obtains two sequences $\left\{a_{n}\right\}_{n \in \mathbb{Z}},\left\{b_{n}\right\}_{n \in \mathbb{Z}}$ belonging to the space $l_{\mathbb{R}}^{\infty}(\mathbb{Z})$ and given by

$$
\begin{aligned}
b_{n}^{2} & =\frac{1}{2} \sum_{j=1}^{g} R_{2 g+2}^{1 / 2}\left(\hat{\mu}_{j}(n)\right) \prod_{k=1, k \neq j}^{g}\left[\mu_{j}(n)-\mu_{k}(n)\right]^{-1}-\frac{1}{4}\left[a_{n}^{2}+a_{n}^{(2)}\right]>0, \\
a_{n} & =\sum_{j=1}^{g} \mu_{j}(n)-\frac{1}{2} \sum_{m=1}^{2 g+2} E_{m}, \\
a_{n}^{(2)} & =\sum_{j=1}^{g} \mu_{j}(n)^{2}-\frac{1}{2} \sum_{m=1}^{2 g+2} E_{m}^{2}, \quad k \in \mathbb{N} .
\end{aligned}
$$

Remark 3.2. The sign of $b_{n}$ is not well defined by this process, and can be arbitrarily chosen. The sequences of the preceding type $a=\left\{a_{n}\right\}_{n \in \mathbb{Z}}, b=\left\{b_{n}\right\}_{n \in \mathbb{Z}}$ are called $g$ gap sequences. They are built on the trace formula of Gelfand-Levitan-Dikii. The lefthand side can be expressed in terms of the Riemann theta function associated with the 
curve $X_{g}$, because they can be regarded as solutions to the Jacobi inversion problem (see [17, Formulae (13a), p. 252, and (13b), p. 253], [6, Formulae (5.9), p. 29, and (5.24), p. 31] and [34, Theorem 7]).

We would like to investigate the difference operator $L=b D^{+}+b D^{-}-a$ with $a, b \in$ $l_{\mathbb{R}}^{\infty}(\mathbb{Z}), b_{n} \neq 0, n \in \mathbb{Z},\left(D^{ \pm} f\right)(n)=f(n \pm 1), n \in \mathbb{Z}, f \in l^{\infty}(\mathbb{Z}), b_{n}^{-}=b_{n-1}, n \in \mathbb{Z}$. The following Jacobi operator $H$, acting on $l^{2}(\mathbb{Z})$ and defined by

$$
H f=L f, \quad f \in \mathcal{D}(H)=l^{2}(\mathbb{Z}),
$$

is the unique self-adjoint extension of $L$ to $l^{2}(\mathbb{Z})$. The two restrictions $H_{ \pm, n_{0}}$ of $H$ to the spaces $l^{2}\left(\left[n_{0}+1,+\infty\right)\right)$ and $l^{2}\left(\left(-\infty, n_{0}-1\right]\right)$ with the Dirichlet condition at $n_{0}$ are now defined by

$$
\begin{aligned}
& H_{+, n_{0}} f=L f, \quad f \in \mathcal{D}\left(H_{+, n_{0}}\right)=\left\{f \in l^{2}\left(\left[n_{0}+1,+\infty\right)\right) \mid f\left(n_{0}\right)=0\right\} . \\
& H_{-, n_{0}} f=L f, \quad f \in \mathcal{D}\left(H_{-, n_{0}}\right)=\left\{f \in l^{2}\left(\left(-\infty, n_{0}-1\right]\right) \mid f\left(n_{0}\right)=0\right\} .
\end{aligned}
$$

We denote by $\sigma(\cdot), \sigma_{\mathrm{ac}}(\cdot), \sigma_{\mathrm{sc}}(\cdot)$ and $\sigma_{\mathrm{p}}(\cdot)$ the spectrum, the absolutely continuous spectrum, the continuous singular spectrum and the point spectrum, respectively [32, pp. 41, 45]. The following result is known.

Theorem 3.3 (see Theorem 4.2 in [6]). Suppose that g-gap sequences $a, b \in l_{\mathbb{R}}^{\infty}(\mathbb{Z})$ with associate operators $H, H_{ \pm, n}$, as previously defined, are given. Then

$$
\sigma(H)=\sigma_{\mathrm{ac}}(H)=\bigcup_{j=1}^{g+1}\left[E_{2 j-1}, E_{2 j}\right], \quad \sigma_{\mathrm{sc}}(H)=\sigma_{\mathrm{p}}(H)=\emptyset
$$

and for each $n$ in $\mathbb{Z}$,

$$
\begin{gathered}
\sigma\left(H_{-, n} \oplus H_{+, n}\right)=\sigma(H) \cup\left\{\mu_{j}(n)\right\}_{1 \leqslant j \leqslant g}, \quad \sigma_{\mathrm{ac}}\left(H_{ \pm, n}\right)=\sigma(H), \\
\sigma_{\mathrm{sc}}\left(H_{ \pm, n}\right)=\emptyset, \quad \sigma_{\mathrm{p}}\left(H_{-, n} \oplus H_{+, n}\right)=\left\{\mu_{j}(n)\right\}_{1 \leqslant j \leqslant g} \cap\left\{\bigcup_{k=1}^{g}\left(E_{2 k-1}, E_{2 k}\right)\right\} .
\end{gathered}
$$

The next result is crucial for our purposes. It is one of the important results in [34] and also in [18]. It is a consequence of the Riemann-Roch Theorem. We outline the proof for the reader's convenience.

Proposition 3.4. Given a finite union of intervals

$$
\Sigma=\left[E_{1}, E_{2}\right] \cup\left[E_{3}, E_{4}\right] \cup \cdots \cup\left[E_{2 g+1}, E_{2 g+2}\right] \quad \text { with } E_{1}<E_{2}<\cdots<E_{2 g+1}<E_{2 g+2},
$$

to any set of points $\left\{\gamma_{j}, j=1, \ldots, g\right\}, \gamma_{j} \in\left[E_{2 j}, E_{2 j+1}\right]$ corresponds a Jacobi operator $T$ unique up to a right composition by a shift operator having

$$
\sigma(T)=\bigcup_{j=1}^{g+1}\left[E_{2 j-1}, E_{2 j}\right] .
$$

Moreover, this operator is almost periodic in the sense of Bohr. 
Proof. In order to prove the existence of the operator $T$ for given $E_{i}, i=1, \ldots, 2 g+2$, we choose $\left\{\gamma_{j}, j=1, \ldots, g\right\}, \gamma_{j} \in\left[E_{2 j}, E_{2 j+1}\right]$. By the Riemann-Roch Theorem, one can find a meromorphic function $\psi_{n}$ on the curve $X_{g}$, associated with the equation

$$
y^{2}=R_{2 g+2}(z)=\prod_{m=1}^{2 g+2}\left(z-E_{m}\right)
$$

unique up a multiplicative factor which has $g$ poles at $\gamma_{j}, j=1, \ldots, g$, and which has a pole of order $n$ at $\infty_{1}$ and a zero of order $n$ at $\infty_{2}$. We normalize $\psi_{n}(P)$, $P=\left(z, \pm R_{2 g+2}(z)^{1 / 2}\right)$ by requiring that the product of the coefficients of $z^{ \pm n}$ in the expansion of $\psi_{n}$ near $\infty_{1}$ and $\infty_{2}$ is 1 . So we can write

$$
\psi_{n}^{ \pm}(z)=\mathrm{e}^{ \pm x_{n}} z^{ \pm n}\left(1+\sum_{s=1}^{\infty} \xi_{s}^{ \pm}(n) z^{-s}\right) .
$$

It follows, from the Riemann-Roch Theorem for example, that $T \psi_{n}(P)=\tilde{\pi}(P) \psi_{n}(P)=$ $z \psi_{n}(P)$, where $T$ is the difference operator associated with the coefficients

$$
\begin{aligned}
& c_{n}=\mathrm{e}^{x_{n}-x_{n+1}}, \\
& v_{n}=\xi_{1}^{+}(n)-\xi_{1}^{+}(n+1),
\end{aligned}
$$

that is $T \psi_{n}=c_{n} \psi_{n+1}+v_{n} \psi_{n}+c_{n-1} \psi_{n-1}$. This gives the existence of $T$. An alternative construction of $T$ can be made by using Lemma 3.1 as in [6, p. 29, Formulae 5.9 and 5.10; p. 31, Formula 5.24]. We choose $\mu_{j}\left(n_{0}\right)=\gamma_{j}, j=1, \ldots, g$, and Lemma 3.1 gives two sequences $a_{n}$ and $b_{n}$ that can be expressed with the help of the theta function of the curve $X_{g}$. This shows that the spectrum $\sigma(T)$ is $E$.

To prove uniqueness, suppose that $J$ is a Jacobi operator with spectrum $\sigma(J)=E$ and $\gamma_{j}, j=1, \ldots, g$, are points as in Proposition 3.4 defining $J$. Then we know $a_{n}$ and $b_{n}$ defining $J: J=b D^{+}+b^{-} D^{-}-a$, with $\sigma\left(H_{-, n} \oplus H_{+, n}\right)=\sigma(H) \cup\left\{\mu_{j}(n)\right\}_{1 \leqslant j \leqslant g}$, so that the $\mu_{j}(n)$ are also known. Suppose that for each $j=1, \ldots, g, \mu_{j}\left(n_{0}\right)=\gamma_{j}$, and consider $\psi\left(P, n, n_{0}\right)$ the stationary Baker-Akhieser function [6]. We denote by $\left(\psi\left(\cdot, n, n_{0}\right)\right)$ the associate divisor. It satisfies [6, p. 17, 3.25 and 3.33]

(1) $J \psi=\tilde{\pi}(P) \psi$

(2) $\left(\psi\left(\cdot, n, n_{0}\right)\right)=\mathcal{D}_{\hat{\mu}(n)}-\mathcal{D}_{\hat{\mu}\left(n_{0}\right)}+\left(n-n_{0}\right)\left(\mathcal{D}_{\infty_{1}}-\mathcal{D}_{\infty_{2}}\right) ;$

(3) $\psi\left(\infty_{1}, n, n_{0}\right) \psi\left(\infty_{2}, n, n_{0}\right)=1$.

From the existence part, we have $\psi\left(\cdot, n, n_{0}\right)=\psi_{n-n_{0}}(\cdot)$. If $\tilde{J}=J-T \circ D^{-n_{0}}$, then $\tilde{J} \psi=0$ and $\tilde{J}=0$ due to the fact that the point spectrum of a non-vanishing Jacobi operator is empty.

Now we give an alternative formulation of Theorem A and Theorem B. 
Theorem C. Let

$$
E=\left[E_{1}, E_{2}\right] \cup\left[E_{3}, E_{4}\right] \cup \cdots \cup\left[E_{2 g-1}, E_{2 g}\right] \cup\left[E_{2 g+1}, E_{2 g+2}\right]
$$

be a given union of intervals and

$$
R_{2 g+2}(z)=\prod_{m=1}^{2 g+2}\left(z-E_{m}\right) .
$$

The two g-gap sequences $\left\{a_{n}\right\}_{n \in \mathbb{Z}}$ and $\left\{b_{n}\right\}_{n \in \mathbb{Z}}$ are periodic if and only if there exist two polynomials $Q(z)$ and $\Delta(z)$ such that $R_{2 g+2}(z) Q(z)^{2}=\Delta(z)^{2}-1$. If this last condition is fulfilled, the period $N$ is given by $N=\operatorname{deg}(Q)+g+1$.

Proof. First we introduce a very important notion, following [17]. We write for short $R(z)=\prod_{m=1}^{2 g+2}\left(z-E_{m}\right)$. The quasimomentum is by definition the meromorphic differential form $\mathrm{d} p$ defined on the curve $X_{g}$ given by

$$
i \mathrm{~d} p=\frac{z^{g}+\sum_{k=0}^{g-1} c_{k} z^{k}}{\sqrt{R(z)}} \mathrm{d} z
$$

normalized by the conditions

$$
\int_{E_{2 i}}^{E_{2 i+1}} \mathrm{~d} p=0, \quad i=1, \ldots, g .
$$

We would like to make precise the link between the quasimomentum and the complex Green function $G(z)$ of $\hat{\mathbb{C}} \backslash E$, with pole at $\infty \in \hat{\mathbb{C}}$, given by (1.1) with the conditions (1.2). The Green function can be described in terms of abelian differentials in the following way. If $R$ is a planar region with $g$ boundary components $\Gamma_{1}, \Gamma_{2}, \ldots, \Gamma_{g}$, its Schottky double $\hat{R}$ is a compact Riemann surface of genus $g$ admitting an anti-conformal involution $\phi$ fixing the boundary $\partial R$. We use the classical notation $\bar{z}=\phi(z)$. For $a, b \in \hat{R}$, let $\omega_{a-b}$ denote the abelian differential of the third kind on $\hat{R}$ with poles of residues 1 and -1 at $a$ and $b$, respectively. If $g(a, z)$ is the Green function of $R$ with logarithmic singularity at $a$, then the differential on $R, \mathrm{~d} g(z, a)+\mathrm{d}^{*} g(z, a)$, extends to an abelian differential of the third kind $\omega_{\bar{a}-a}[\mathbf{1 1}]$. It is crucial here that if $\Gamma_{i}=\left[E_{2 i-1}, E_{2 i}\right], 1 \leqslant i \leqslant g+1$, then the Schottky double $\hat{R}$ is the hyperelliptic curve $X_{g}$ associated with the equation $y^{2}=\prod_{i=1}^{2 g+2}\left(z-E_{i}\right)[\mathbf{1 1}]$, where it was shown that

$$
\begin{aligned}
G(z) & =\int_{E_{1}}^{z} i \mathrm{~d} p \\
& =\int_{E_{1}}^{z} \omega_{\infty+, \infty-\cdot}
\end{aligned}
$$

Now the normalization conditions on the quasimomentum $\mathrm{d} p$ are exactly those in (1.2) or (2.5). But Krichever [18] proved that the polynomial condition on $R(z)$ in Theorem $\mathrm{C}$ is equivalent to the conditions

$$
\frac{1}{2 \pi} \int_{E_{2 j}}^{E_{2 j+1}} \mathrm{~d} p=\frac{m_{k}}{N}, \quad m_{k} \in \mathbb{N}
$$


and this is exactly the condition (2.6). The final conclusion at this point is that the existence of a periodic Jacobi matrix that is real, symmetric and $N$-periodic having a union of intervals $E$ as spectrum is equivalent to the fact that the $N$ th Chebyshev polynomial oscillates exactly $N$ times on $E$.

In the rest of this section, we give more details on the last connection involved in the proof of Theorem C.

\subsection{Green functions, capacities and a new look at a theorem of Walsh}

Definition 3.5. A compact set $E=\left[E_{1}, E_{2}\right] \cup\left[E_{3}, E_{4}\right] \cup \cdots \cup\left[E_{2 n-1}, E_{2 n}\right]$ of the real line is calibrated if the complex Green function $G(z)$ of $\hat{\mathbb{C}} \backslash E$ with pole at infinity given by (1.1) satisfies the conditions (2.5) and (2.6)

$$
\int_{E_{2 k}}^{E_{2 k+1}} \sqrt{Q(t)} \mathrm{d} t=0, \quad k=1,2, \ldots, n-1,
$$

and

$$
\int_{E_{2 k-1}}^{E_{2 k}} \sqrt{Q(t)} \mathrm{d} t= \pm \frac{r_{k} \pi \mathrm{i}}{N}, \quad k=1,2, \ldots, n
$$

where

$$
Q(t)=\frac{\left(t-c_{1}\right)^{2}\left(t-c_{2}\right)^{2} \cdots\left(t-c_{n-1}\right)^{2}}{\left(t-E_{1}\right)\left(t-E_{2}\right) \cdots\left(t-E_{2 n-1}\right)\left(t-E_{2 n}\right)}=h(t)^{2}
$$

and $r_{1}, \ldots r_{n}, N \in \mathbb{N}, r_{1}+\cdots+r_{n}=N$.

We observe that if a compact $E$ is calibrated with respect to $r_{1}, r_{2}, \ldots, r_{n}, N$, then it is also calibrated with respect to $k r_{1}, k r_{2}, \ldots, k r_{n}, k N, k \in \mathbb{N}^{*}$. Now we give an illustration of this definition.

Theorem 3.6. If $E$ is calibrated, then a sequence of integers $\left(m_{k}\right)_{k \in \mathbb{N}}$ exists such that the $m_{k}$ th Chebyshev polynomial is

$$
T_{m_{k}}(z)=2 \mathcal{C}(E)^{m_{k}} \cosh \left(m_{k} G(z)\right) .
$$

Proof. We take the determination of $\sqrt{Q(t)}$ that corresponds to the simply connected domain $\hat{\mathbb{C}} \backslash]-\infty, E_{2 n}$ ] that is positive for real $x>E_{2 n}$. If $E$ is calibrated with respect to $r_{1}, r_{2}, \ldots, r_{n}, N$, with $r_{1}+r_{2}+\cdots+r_{n}=N$, the function

$$
T_{N}(z)=M_{N} \cosh \left(N \int_{E_{2 n}}^{z} \sqrt{Q(t)} \mathrm{d} t\right)
$$

is an entire function which behaves on circles $\{|z|=r\}$ like

$$
T_{N}(z) \sim r^{N}, \quad r \mapsto \infty .
$$

$T_{N}(z)$ is then a polynomial and it is the Chebyshev polynomial of degree $N$ of $E$, the constant $M_{N}$ is such that $T_{N}(z)$ is a monic polynomial. As we observed, the function

$$
T_{k N}(z)=M_{k N} \cosh \left(k N \int_{E_{2 n}}^{z} \sqrt{Q(t)} \mathrm{d} t\right)
$$


is also the Chebyshev polynomial of $E$ of degree $k N$ if the constant $M_{k N}$ is such that $T_{k N}(z)$ is a monic polynomial. Now, if $t_{N}(z)$ is the $N$ th Chebyshev polynomial of the interval $[-2,2]$, then

$$
T_{k N}(z)=2^{-k} M_{N}^{k} t_{k}\left(\cosh \left(2 \frac{T_{N}(z)}{M_{N}}\right)\right)=z^{k N}+\cdots,
$$

and $T_{k N}(z)$ oscillates $k N$ times between $\pm M_{N}^{k}$ on $E$ and so it is the $(k N)$ th Chebyshev polynomial of $E$. It follows that

$$
M_{k N}=2^{1-k} M_{N}^{k}, \quad k \in \mathbb{N},
$$

or

$$
\mathcal{C}(E)=\left(\frac{1}{2} M_{N}\right)^{1 / N}
$$

giving

$$
T_{k N}(z)=2 \mathcal{C}(E)^{k N} \cosh \left(k N \int_{E_{2 n}}^{z} \sqrt{Q(t)} \mathrm{d} t\right) .
$$

To compare Equations (3.6) and (3.7), we use the classical identity

$$
t_{N}(2 \cosh (\theta))=2 \cosh (N \theta)
$$

to transform the formula (3.6) into

$$
T_{k N}(z)=\frac{1}{2} M_{k N} t_{N}\left(2 \cosh \left(N \int_{E_{2 n}}^{z} \sqrt{Q(t)} \mathrm{d} t\right)\right)
$$

and by (3.5) and (3.8), we obtain the formula (3.7). This proves the theorem.

For the special case of calibrated $E$, the theorem gives an exact relation between the complex Green function of $\hat{\mathbb{C}} \backslash E$ and a special family of Chebyshev polynomials of $E$. In this case, we have a precise formulation of a result of Widom [38, Formulae 2.5 and 2.7] which asserts that

$$
\lim _{n \rightarrow \infty} T_{n}(z) \mathcal{C}(E)^{-n} \Phi(z)^{-n}=1
$$

uniformly on compact sets of the complement of $E$. Here $\Phi(z)$ is the exponential of the Green function of the complement of $E$. Moreover, it has been proved by Walsh [37, pp. $72,73,157,158]$ that if $G(z)$ is the Green function with pole at infinity of an unbounded region of finite connectivity $\Omega$, whose boundary consists of a finite number of non-intersecting analytic Jordan curves, then there exist points $z_{1}, z_{2}, \ldots$ in the complement $E$ of $\Omega$ such that

$$
\lim _{n \rightarrow \infty}\left|\left(z-z_{1}\right)\left(z-z_{2}\right) \cdots\left(z-z_{n}\right)\right|^{1 / n}=\mathcal{C}(E) \exp (G(z)),
$$

the convergence being uniform on compact sets of $\Omega$. From (3.6), (3.7) and (3.8) we have for calibrated $E$ a precise statement of Walsh's result.

Before continuing further, we wish to recall some definitions that will be needed. Given a bounded set $\mathcal{E}$ in the complex plane, we denote by $\mathcal{E}(r)$ the $r$-neighbourhood of $\mathcal{E}$. 


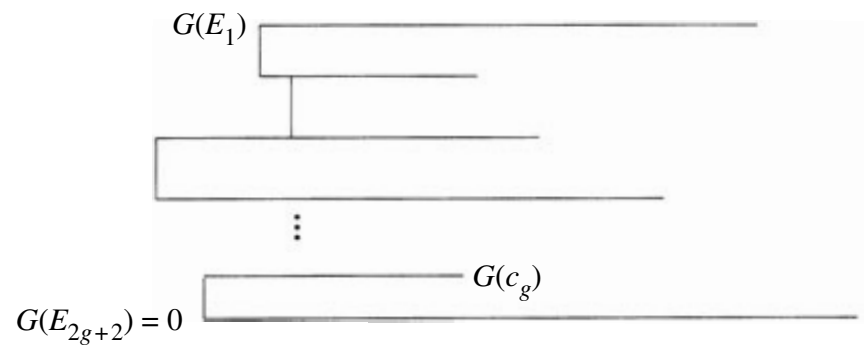

Figure 1 .

Definition 3.7. If $\mathcal{E}_{1}$ and $\mathcal{E}_{2}$ are two bounded sets in $\mathbb{C}$, the difference between $\mathcal{E}_{1}$ and $\mathcal{E}_{2}$ is the smallest $r$ such that $\mathcal{E}_{1}(r)$ contains $\mathcal{E}_{2}$ and $\mathcal{E}_{2}(r)$ contains $\mathcal{E}_{1}$.

We will denote this difference by $\delta\left(\mathcal{E}_{1}, \mathcal{E}_{2}\right)$. It is small if and only if $\mathcal{E}_{1}$ and $\mathcal{E}_{2}$ are (almost) superimposable. If $\mathcal{E}$ is a compact set and $\left(\mathcal{E}_{\nu}\right)$ a family of sets such that $\delta\left(\mathcal{E}, \mathcal{E}_{\nu}\right)$ tends to zero as $\nu$ tends to $\infty$, we will say simply that $\left(\mathcal{E}_{\nu}\right)$ tends to $\mathcal{E}$. The following lemma is important for our needs [7].

Lemma 3.8. If $\left(\mathcal{E}_{\nu}\right)$ tends to $\mathcal{E}$, then for a fixed $n$, the Chebyshev polynomials $T_{n}\left(z, \mathcal{E}_{\nu}\right)$ tend to the Chebyshev polynomials $T_{n}(z, \mathcal{E})$ as $\nu$ tends to $\infty$, uniformly on $\mathcal{E}$.

The main result of this section is the following approximation theorem, the first part of which was proved implicitly by Robinson in [29]. The main idea here is to exhibit the connection between the difference operators attached to Jacobi matrices and the Carathéodory Kernel Theorem for conformal mappings. The reason is that, as in the continuous case [20], the Floquet exponent is related to the Schwarz-Christoffel formula, which is in turn related to the complex Green function.

Theorem 3.9. Let $E=\left[E_{1}, E_{2}\right] \cup\left[E_{3}, E_{4}\right] \cup \cdots \cup\left[E_{2 g+1}, E_{2 g+2}\right]$ be a finite union of closed intervals. There exists a family of calibrated sets $E_{\nu}=\left[E_{1, \nu}, E_{2, \nu}\right] \cup\left[E_{3, \nu}, E_{4, \nu}\right] \cup$ $\cdots \cup\left[E_{2 g+1, \nu}, E_{2 g+2, \nu}\right]$ tending to $E$. Consequently, each real and symmetric Jacobi matrix whose spectrum is a finite union of disjoint closed intervals is a limit of real, symmetric and periodic Jacobi matrices.

Proof. For a given $E=\left[E_{1}, E_{2}\right] \cup\left[E_{3}, E_{4}\right] \cup \cdots \cup\left[E_{2 g+1}, E_{2 g+2}\right]$ and $c_{j} \in\left[E_{2 j}, E_{2 j+1}\right]$, $1 \leqslant j \leqslant g$, the function $z \mapsto w=G(z)$ maps the upper half-plane onto a polygon of the type in Figure 1.

From the solution of the Jacobi inversion problem, there is a unique set

$$
\left\{c_{j} \in\left[E_{2 j}, E_{2 j+1}\right], 1 \leqslant j \leqslant g\right\}
$$

such that the polynomial

$$
h(\zeta)=\zeta^{g}+h_{g-1} \zeta^{g-1}+\cdots+h_{0}=\left(\zeta-c_{1}\right)\left(\zeta-c_{2}\right) \cdots\left(\zeta-c_{g}\right)
$$

satisfies the system (1.2). In this situation,

$$
G\left(E_{2 j}\right)=G\left(E_{2 j+1}\right), \quad 1 \leqslant j \leqslant g, \quad G\left(E_{1}\right)=\mathrm{i} \pi, \quad G\left(E_{2 g+2}\right)=0 .
$$




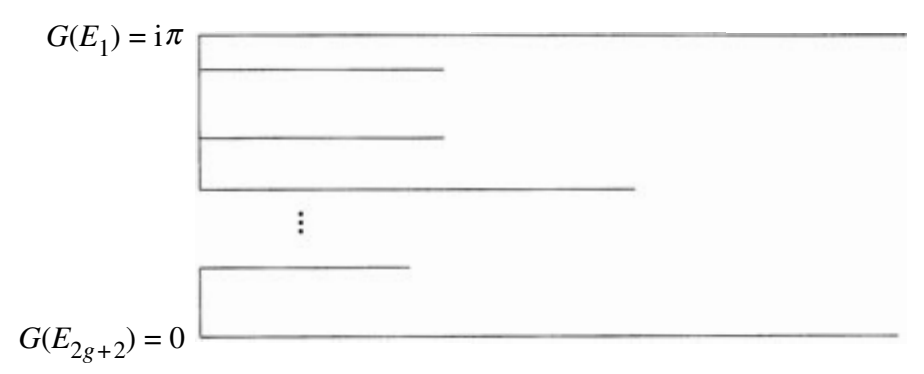

Figure 2.

The map $z \mapsto w=G(z)$ sends the upper half-plane onto the polygon $\Gamma$ of the form in Figure 2.

The calibration of $E$ means that the slits have heights of the form $q / r \pi, q, r \in \mathbb{N}^{*}$. If a finite union of intervals $E=\left[E_{1}, E_{2}\right] \cup\left[E_{3}, E_{4}\right] \cup \cdots \cup\left[E_{2 g+1}, E_{2 g+2}\right]$ is given, its image $\Gamma$ under the map $z \mapsto w=G(z)$ with $G(z)$ satisfying (1.2) does not necessarily satisfy the second condition in the definition of calibration. But we can find a sequence $\left(\Gamma_{\nu}\right)$ of polygons tending to $\Gamma$ as $\nu$ tends to $\infty$ in the sense that $\lim _{\nu \rightarrow \infty} \delta\left(\Gamma, \Gamma_{\nu}\right)=0$, where $\left(\Gamma_{\nu}\right)$ is bounded horizontally by the two half $\operatorname{lines} \operatorname{Im} w=0, \operatorname{Im} w=\mathrm{i} \pi$ and vertically by the imaginary axis. Finally, it has $g$ horizontal slits with heights equal to rational multiples of $\pi$. From the definition of the difference $\delta\left(\Gamma, \Gamma_{\nu}\right)$ we see that $\Gamma$ is the kernel of the family $\left(\Gamma_{\nu}\right)$, in the sense that $\Gamma$ is the largest domain $D$ containing $\infty$, and each compact set $K$ of $D$ is contained in $\Gamma_{\nu}$ for $\nu \geqslant \nu_{0}(K)$. By the Riemann mapping theorem, there is a unique conformal mapping $g_{\nu}$ of the slit domain $\Gamma_{\nu}$ onto the upper half-plane, which, by another theorem of Carathéodory, can be extended continuously and homeomorphically up to the boundary. The image $g_{\nu}([0, \mathrm{i} \pi])$ is a calibrated set $E_{\nu}=\left[E_{1, \nu}, E_{2, \nu}\right] \cup\left[E_{3, \nu}, E_{4, \nu}\right] \cup \cdots \cup\left[E_{2 g+1, \nu}, E_{2 g+2, \nu}\right]$ tending to $E$ as $\nu$ tends to $\infty$, by the Carathéodory Kernel Theorem [13]. We have thus proved that any finite union $E$ of closed intervals can be approximated in the difference topology by a calibrated finite union $E_{\nu}$ of closed intervals. By (3.8), any Chebyshev polynomial $T_{n}(z, E)$ is a limit of a uniformly convergent sequence on $E$ of Chebyshev polynomials $T_{n}\left(z, E_{\nu}\right)$ of calibrated $E_{\nu}$.

To conclude this proof, we add one more remark. The universal covering of $\hat{\mathbb{C}} \backslash E$ and $\hat{\mathbb{C}} \backslash E_{\nu}$ is the upper half-plane $\mathcal{H}$. From [13, Theorem 1], the universal covering maps $\eta_{\nu}: \mathcal{H} \mapsto \mathbb{C} \backslash E_{\nu}$ converge uniformly on compact sets of $\mathcal{H}$ to the universal covering map $\eta: \mathcal{H} \mapsto \mathbb{C} \backslash E$.

To close this section, we wish to point out a relation between the resolvent of a real symmetric and periodic Jacobi matrix of spectrum $E$ and the complex Green function of $\hat{\mathbb{C}} \backslash E$. We write

$$
\begin{aligned}
G^{\prime}(\lambda) \mathrm{d} \lambda & =\mathrm{id} p(\lambda)=\omega_{\infty+, \infty-}^{(3)}(\lambda) \\
& =\frac{\prod_{j=1}^{g}\left(\lambda-\lambda_{j}\right)}{\sqrt{R_{2 g+2}}(\lambda)} \mathrm{d} \lambda=\frac{P(\lambda)}{\sqrt{R_{2 g+2}}(\lambda)} \mathrm{d} \lambda
\end{aligned}
$$


where $\omega_{\infty+, \infty-}^{(3)}(\lambda)$ is the normalized differential of the third kind with poles at $\infty+$, $\infty-$ and $\lambda_{j} \in\left[E_{2 j}, E_{2 j+1}\right], j=1, \ldots, g$. We choose $\mu_{j}\left(n_{0}\right)=\lambda_{j}, j=1, \ldots, g$. By (3.3), to each $\mu_{j}\left(n_{0}\right), j=1, \ldots, g$, there corresponds a unique Jacobi operator $H^{\left(n_{0}\right)}$ of spectrum $\Sigma=\bigcup_{j=0}^{g}\left[E_{2 j+1}, E_{2 j+2}\right]$. Denoting the Green function (resolvent) of $H^{\left(n_{0}\right)}$ by $G^{\left(n_{0}\right)}(z, n, m)$, the diagonal Green function is given by $[\mathbf{6}]$

$$
G^{\left(n_{0}\right)}\left(z, n_{0}, n_{0}\right)=\frac{\prod_{j=1}^{g}\left[z-\mu_{j}\left(n_{0}\right)\right]}{\sqrt{R_{2 g+2}(z)}} .
$$

In other words, we have the identity

$$
G^{\left(n_{0}\right)}\left(z, n_{0}, n_{0}\right)=G^{\prime}(z) .
$$

Now, for every $n, \mu_{j}(n)$ is defined from $\mu_{j}\left(n_{0}\right)$, as is $(G(z, n, n))_{n}$.

\subsection{Example}

For a fixed $a>1$ the compact set $\Sigma_{a}=[-a-1,-a+1] \cup[a-1, a+1]$ is mapped under $z \mapsto z^{2}$ onto the interval $\left[(a-1)^{2},(a+1)^{2}\right]$ of capacity $a$. By the transfer theorem, the capacity of $\Sigma_{a}$ is $\sqrt{a}$. As is shown in [35], a Jacobi matrix $J$ of period 2 and whose spectrum is exactly $\Sigma_{a}$ is, for instance, given by

$$
J_{n, n}=a_{n}=a_{n+2}, \quad J_{n, n+1}=J_{n+1, n}=b_{n}=b_{n+2},
$$

with

$$
\begin{gathered}
a_{0}+a_{1}=0, \quad b_{0} b_{1}=a, \\
a^{2}+1=a_{0}^{2}+b_{0}^{2}+b_{1}^{2} .
\end{gathered}
$$

This shows again that the capacity of $\Sigma_{a}$ is $\sqrt{b_{0} b_{1}}=\sqrt{a}$. Furthermore, the complex plane slit along $[-1, \alpha] \cup[\beta, 1]$ is conformal to the complex plane slit along $[-1,-a] \cup[a, 1]$, where

$$
a=\frac{1-k^{\prime}}{1+k^{\prime}}, \quad k^{2}+k^{2}=1, \quad k^{2}=\frac{2(\beta-\alpha)}{(1+\alpha)(1+\beta)} .
$$

\section{References}

1. N. I. Achieser, Sur les polynômes de Tchebyscheff pour deux segments, C. R. Acad. Sci. Paris 191 (1930), 754-756.

2. N. I. ACHIESER, Theory of approximation (Frederick Ungar Publishing, New York, 1956).

3. I. Bendixon, Sur les racines d'une équation fondamentale, Acta Math. 25 (1902), 359366 .

4. R. BOAS, Entire functions (Academic, 1954).

5. P. Borwein, The arclength of the lemniscate $\{|p(z)|=1\}$, Proc. Am. Math. Soc. 123 (1995), 797-799.

6. W. Bulla, F. Gesztesy, H. Holden and G. Teschl, Algebro-geometric quasi-periodic finite-gap solutions of the Toda and Kac-van Moerbeke hierarchies, Mem. Am. Math. Soc. 135 (1998), 1-79. 
7. G. Calugareanu, Sur les polynômes de Tchebichef d'un ensemble plan borné et fermé, Bull. Sci. Math. 69 (1945), 75-81.

8. B. C. CARlson And J. TODD, Zolotarev's first problem-the best approximation by polynomials of degree $\leqslant n-2$ to $x^{n}-n \sigma x^{n-2}$ in $[-2,2]$, Aequat. Math. 26 (1983), 1-33.

9. A. CUYT, K. Driver And D. S. Lubinsky, On the size of lemniscates of polynomials in one and several variables, Proc. Am. Math. Soc. 124 (1996), 2123-2136.

10. A. Eremenko and W. Hayman, On the length of Lemniscates, Michigan Math. J. 46 (1999), 409-415.

11. Th. FAlliero And A. SebBar, Capacité de la réunion de trois intervalles et fonctions thêta de genre 2, J. Math. Pures Appl. 80 (2001), 409-443.

12. M. Fekete, Über den transfiniten Durchmesser ebener Punktmengen, Erste Mitteilung, Math. Z. 32 (1930), 108-114.

13. D. A. HejhaL, Universal covering maps for variable regions, Math. Z. 137 (1974), 7-20.

14. A. Hirsch, Sur les racines d'une équation fondamentale, Acta Math. 25 (1902), 367-370.

15. F. Hirzebruch, Th. Berger and R. Jung, Manifolds and modular forms, in Aspects of mathematics (Max-Planck Institut für Mathematik, Bonn, 1992).

16. E. L. INCE, Ordinary differential equations (Dover, New York, 1956).

17. I. M. Krichever, The Peierls model, Funkts. Analysis Prilozhen 16 (1982), 10-26.

18. I. M. KRICHEVER, Nonlinear equations and elliptic curves, Current problems in mathematics, vol. 23, pp. 79-136 (Itogi Nauki i Tekhniki, Akad. Nauk SSSR, Vsesoyuz. Inst. Nauchn. i Tekhn. Inform., Moscow, 1983).

19. I. M. KRICHEVER, The spectral theory of finite-gap nonstationary Schrödinger operators, The nonstationary Peierls model, Funkts. Analysis Prilozhen 20 (1986), 42-54.

20. J. Moser, Integrable Hamiltonian systems and spectral theory (Academia Nazionale dei Lincei, Fermi Lecture, Pisa, 1981).

21. P. B. NAĬMAN, On the theory of periodic and limit-periodic Jacobi matrices, Sov. Math. Dokl. 3 (1962), 383-385.

22. I. V. Ostrovski, F. B Pakovitch and M. G. ZaidenberG, A remark on complex polynomials of least deviation, Int. Math. Res. Not. 14 (1996), 699-703.

23. F. B. Pakovitch, Elliptic polynomials, Russ. Math. Surv. 50 (1995), 1292-1294.

24. F. Peherstorfer, On Bernstein-Szegö orthogonal polynomials on several intervals, II, Orthogonal polynomials with periodic recurrence coefficients, J. Approx. Theory 64 (1991), 123-161.

25. F. Peherstorfer, On orthogonal and extremal polynomials on several intervals, J. Comput. Appl. Math. 48 (1993), 187-205.

26. F. Peherstorfer, Minimal polynomials for compact sets of the complex plane, Constr. Approx. 12 (1996), 481-488.

27. F. Peherstorfer, Deformation of minimal polynomials and approximation of several intervals by an inverse polynomial mapping, J. Approx. Theory 111 (2001), 180-195.

28. C. H. Pommerenke, Über die Kapazität ebener Kontinuen, Math. Annln 141 (1960), 143-152.

29. R. M. Robinson, Conjugate algebraic integers in real point sets, Math. Z. 84 (1964), 415-427.

30. R. M. Robinson, Intervals containing infinitely many sets of conjugate algebraic units, Ann. Math. 80 (1964), 411-428.

31. A. Schinzel, Selected topics on polynomials (University of Michigan Press, Ann Arbor, MI, 1982).

32. G. TESCHL, Jacobi operators and completely integrable nonlinear lattices, Mathematical Surveys and Monographs, vol. 72 (American Mathematical Society, Providence, RI, 2000).

33. P. VAN Moerbeke, The spectrum of Jacobi matrices, Invent. Math. 37 (1976), 45-81. 
34. P. VAn MoERBeke AND D. Mumford, The spectrum of difference operators and algebraic curves, Acta Math. 143 (1979), 93-154.

35. S. Venakides, P. Deift and R. ObA, The Toda shock problem, Commun. Pure Appl. Math. 44 (1991), 1171-1242.

36. A. P. Veselov, Integrable maps, Russ. Math. Surv. 46 (1991), 1-51.

37. J. L. WALSH, Interpolation and approximation by rational functions in the complex plane, 3rd edn, American Mathematical Society Colloquium Publications, vol. 20 (American Mathematical Society, Providence, RI, 1960).

38. H. WIDOM, Extremal polynomials associated with a system of curves in the complex plane, Adv. Math. 3 (1969), 127-232. 